\title{
PZT Sensor Array for Local and Distributed Measurements of Localized Cracking in Concrete
}

Article in Smart Materials and Structures · June 2018

DOI: 10.1088/1361-665X/aaca4d

CITATIONS

3 authors:

Arun Narayanan

Indian Institute of Technology Hyderabad

10 PUBLICATIONS 27 CITATIONS

SEE PROFILE

Kolluru Subramaniam

Indian Institute of Technology Hyderabad

130 PUBLICATIONS $\mathbf{1 , 4 8 1}$ CITATIONS

SEE PROFILE
READS

67

Amarteja Kocherla

Indian Institute of Technology Hyderabad

5 PUBLICATIONS $\mathbf{4}$ CITATIONS

SEE PROFILE

Some of the authors of this publication are also working on these related projects:

Failure investigation in Clay Brick Masonry with Soft Brick View project

Damage Assesment of Concrete Elements using PZT Based Sensors View project 
ACCEPTED MANUSCRIPT

\section{PZT Sensor Array for Local and Distributed Measurements of Localized Cracking in Concrete}

To cite this article before publication: Arun Narayanan et al 2018 Smart Mater. Struct. in press https://doi.org/10.1088/1361-665X/aaca4d

\section{Manuscript version: Accepted Manuscript}

Accepted Manuscript is "the version of the article accepted for publication including all changes made as a result of the peer review process, and which may also include the addition to the article by IOP Publishing of a header, an article ID, a cover sheet and/or an 'Accepted

Manuscript' watermark, but excluding any other editing, typesetting or other changes made by IOP Publishing and/or its licensors"

This Accepted Manuscript is @ 2018 IOP Publishing Ltd.

During the embargo period (the 12 month period from the publication of the Version of Record of this article), the Accepted Manuscript is fully protected by copyright and cannot be reused or reposted elsewhere.

As the Version of Record of this article is going to be / has been published on a subscription basis, this Accepted Manuscript is available for reuse under a CC BY-NC-ND 3.0 licence after the 12 month embargo period.

After the embargo period, everyone is permitted to use copy and redistribute this article for non-commercial purposes only, provided that they adhere to all the terms of the licence https://creativecommons.org/licences/by-nc-nd/3.0

Although reasonable endeavours have been taken to obtain all necessary permissions from third parties to include their copyrighted content within this article, their full citation and copyright line may not be present in this Accepted Manuscript version. Before using any content from this article, please refer to the Version of Record on IOPscience once published for full citation and copyright details, as permissions will likely be required. All third party content is fully copyright protected, unless specifically stated otherwise in the figure caption in the Version of Record.

View the article online for updates and enhancements. 


\section{PZT Sensor Array for Local and Distributed Measurements}

\section{of Localized Cracking in Concrete}

${ }^{a}$ Department of Civil Engineering, Indian Institute of Technology Hyderabad, Hyderabad, TS 502285, INDIA

\section{Abstract}

An application is developed with surface mounted Lead Zirconate Titanate (PZT) patches for sensing damage in the form of a stress-induced crack in a concrete substrate. A localized crack is introduced in a controlled manner using a fracture test. Full-field displacements obtained using digital image correlation are used for crack penetration and crack width measurements. Electrical impedance (EI) measurements are obtained from the individual PZT patches, which are attached at different locations relative to the crack. Stress wave transmission measurements are performed using the PZT patches as actuator-receiver (AR) pairs. The EI measurements indicate that small, quantifiable changes in the mechanical impedance of the substrate are experienced by the PZT patch in the vicinity of the localized crack, which sensitively detect crack initiation. The stress wave-based measurements are very sensitive to the presence of physical discontinuity created by a localized crack in the stress wave path. A measure of stress wave attenuation, the attenuation factor is developed, to quantify the measured changes in the stress wave produced by the physical discontinuity in concrete upon

${ }^{1}$ Corresponding Author: Professor, Department of Civil Engineering, Indian Institute of Technology Hyderabad, Hyderabad, TS 502205, INDIA. ph.:+91-402-301-6093, email: kvls@iith.ac.in 
unloading a stress-induced crack. The physical discontinuity due to a stress-induced crack opening on the order of $10 \mu \mathrm{m}$ can be detected from the measured changes in the attenuation factor. The physical discontinuity in the concrete associated with a stress-induced crack opening on the order of $100 \mu \mathrm{m}$ produces a complete attenuation of the stress wave of $120 \mathrm{kHz}$. The combined use of PZT patches in the EI and the AR modes can be used to detect local changes close to a PZT patch and it allows distributed sensing over the entire volume of a structural element.

\section{Introduction}

PZT is a piezoelectric material, which is being used for developing economical methods for continuous damage assessment in structures. PZT exhibits a coupled electromechanical (EM) response; surface charges are produced when mechanical strain is induced and strain when electrical potential is applied. PZT-based sensors offer a significant potential for continuously monitoring the development and progression of internal damage in structures.

Several damage detection strategies have been developed using PZT patches attached to a substrate [Song et al. (2008), Zhu and He (2011), Lim et al (2012), Lu et al. (2013), Rucka and Wilde (2013), Divsholi and Yang (2014), Narayanan and Subramaniam (2016a, b), Liang et al. (2016), Huo et al. (2017), Ai et al. (2017)]. The use of PZT patches has been primarily focused on metallic and composite structures with very limited application to concrete. The use of PZT patches in concrete structures is still evolving.

The electrical impedance (EI) obtained from the measured electrical response of a PZT patch at multiple frequencies depends on the electromechanical (EM) response of the PZT material, the geometry of the patch and the boundary conditions. The electrical impedance measurements from a PZT patch bonded to a substrate depend on the EM response of the 
coupled system. When a PZT patch is attached to a substrate, the dynamic motion of the PZT patch in response to an applied electrical potential depends on the dynamic mechanical impedance to its motion provided by the substrate, the mechanical impedance [Liang et al. (1994), Giurgiutiu et al. (1999), S. Park et al. (2006), Na and Lee (2012), Narayanan et al. (2017)]. The use of a PZT patch to infer about the level of damage in the substrate requires interpreting the coupled EM response of the PZT patch attached to the substrate. Understanding the response of PZT patch attached to a concrete substrate is still evolving. Changes are registered in EI measurements due to formation of cracks well in advance of failure [Park et al. (2000)]. The EM impedance (EMI) derived from the electrical measurements on PZT patches attached to a concrete substrate sensitively detect changes in the local material compliance produced by distributed damage in the vicinity of the sensor [Narayanan and Subramaniam $(2016 \mathrm{a}, \mathrm{b})]$. For a PZT patch attached to a concrete substrate, its motion at a given frequency is directly influenced by a zone of influence, which represents the finite volume of material. The EMI measurements from a concrete substrate are shown to be sensitive to incipient distributed damage in the material within the zone of influence [Narayanan et al. (2018)]. A smaller zone of influence and higher sensitivity to local changes increases at higher frequencies. The EI measurements therefore provide a local measure of damage in the vicinity of the sensor.

The coupled constitutive electro-mechanical response of piezoelectric material allows a PZT patch to be used as an actuator for generating stress waves in the substrate material and as a receiver for sensing stress waves. The PZT patches are used as actuator/receiver (AR) pairs for generating and receiving stress waves. In the distributed sensing mode, damage in the material is inferred through changes in the elastic waves which propagate through the bulk material [Jung et al. (2002), Aggelis and Shiotani (2007), Marani et al. (2014)]. Presence of cracks in the wave propagation has been shown to significantly alter the wave characteristics 
71 [Lu et al. (2013), Watanabe et al. (2014), Kee and Nam (2015), Luo et al. (2016)]. Most of the studies of PZT-based distributed monitoring were reported on metallic structures. The condition monitoring of concrete infill in fiber reinforced polymer tubes and failure in concrete and composite structures has been monitored using PZT sensor arrays [Xu et al. (2017), Memmolo et al. (2016), Divsholi and Yang (2014), Lu et al. (2013)]. Most of the researchers used time of flight and decrease in wave energy to assess changes in the material characteristics.

In concrete, damage initiation takes place in the form of distributed micro cracks, which eventually coalesce to form localized cracks. Cracks in concrete are associated with cohesive crack bridging stresses. There may be significant degradation of the capacity of the structure by the time of appearance of visible cracking on the surface of a concrete structure. Initiation of early intervention measures, which can effectively increase the service life of the structure require early detection of damage. Methods to detect ineipient damage in the form of micro cracks are required to provide effective methods of monitoring structural health and service life performance of structures. Procedures for locating cracks in concrete and for assessing the depth of opening of these cracks is critical for evaluating the degradation of concrete structures.

Localized sensing methodology based on EI technique provides information about changes in the local material, which produce changes in its compliance in the vicinity of PZT patch. Any damage located away from the sensing range of a PZT patch would not be detected in its EM signature. In concrete structures where large volume of material has to be monitored a large number of PZTs is required for local sensing. Sensing methodology using pairs of PZT patches for monitoring changes in the stress wave can be used for distributed sensing over a larger region. Local sensing technique (EI measurements) can be employed to detect the damage in the vicinity of the PZT patches while distributed sensing using PZT sensor arrays can be used to monitor the location and the magnitude of damage in a region. The concept of 
array of sensors which combines both local and distributed sensing using minimum number of sensors provides detailed interpretation about the damage.

A sensing scheme using an array of PZT sensors for combined local and distributed damage monitoring is developed. Surface mounted PZT patches are used for continuous local monitoring of concrete and obtaining the information related to damage in the vicinity of the patch. Additionally, the PZT sensor array is used for monitoring damage in the actuatorreceiver (AR) mode. The results of an experimental evaluation involving the use of a fracture test specimen are presented and issues related to the development of proposed system are evaluated for the case of localized damage in the form of a crack in concrete. The sensitivity of the EM impedance-based local measurements and the through-transmission measurements to a localized crack in concrete is evaluated.

\section{Background}

PZT patches are made of piezoceramic materials, which have high electro-mechanical coupling properties. The electrical impedance (inverse of admittance) signature of the PZT patch can be measured when it is excited with an alternating electrical potential. The electrical impedance is a complex number consisting of real and imaginary parts, and is determined as the ratio of the current to the applied voltage. The electrical conductance response (real part of electrical admittance) of a PZT patch (20 mm x $20 \mathrm{~mm}$ x $1 \mathrm{~mm}$ size $)$ when an alternating potential excitation of $1 \mathrm{~V}$ amplitude over a range of frequencies between $10 \mathrm{kHz}$ and $500 \mathrm{kHz}$, is shown in Figure 1. The peaks in the conductance response can be identified with the resonant modes of the PZT patch [Liang et al. (1994), Xu and Liu (2002]. The mechanical and the electromechanical resonances happen at the same frequency in a piezoelectric material because of the electro-mechanical coupling. The electro-mechanical resonance frequencies of 
120

121

122

10

the PZT patch depends on the mechanical resonances which in turn depends on geometry of the patch [Giurgiutiu (2001)].

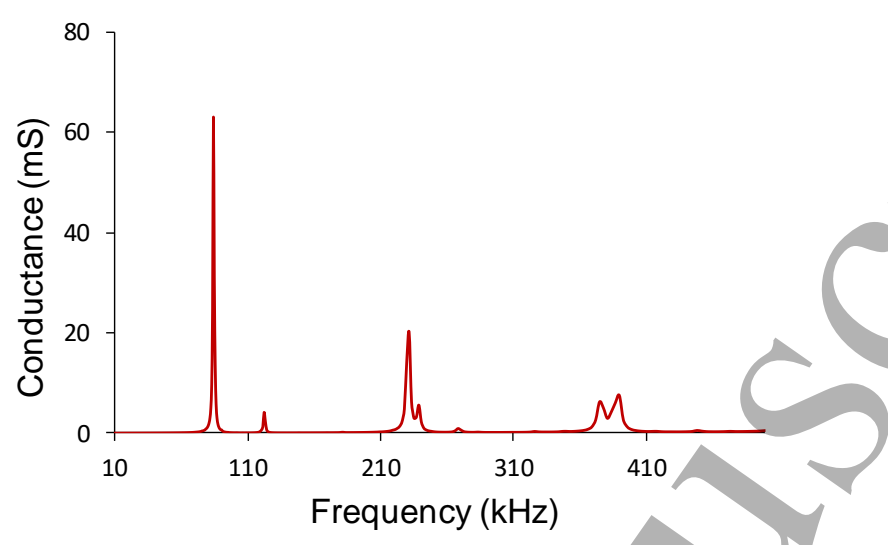

Figure 1. Electrical conductance spectrum of a free PZT patch (patch size: $20 \mathrm{~mm}$ x $20 \mathrm{~mm}$ $\mathrm{x} 1 \mathrm{~mm})$

The EI response of a PZT patch attached to the substrate subjected to an applied electrical potential depends on the dynamic impedance to its motion from the substrate. The resistance to the motion of a PZT patch from the surrounding elastic medium is expressed as the mechanical impedance. Most approaches for modeling the PZT patch-structure interaction have varied in the degree of sophistication in representing the motion of the PZT patch and the structure. The first systematic attempt to model the PZT patch-structure interaction was presented by Liang et al. (1994) using a PZT actuator driven one-degree-of-freedom springmass-damper system. Subsequently, for a PZT patch, an effective 1-D approach was found to give a better representation of the dynamic response of the PZT considering in-plain motion of the PZT coupled to an elastic substrate [Bhalla and Soh (2004)]. The frequency dependent complex admittance response of the PZT patch, $\bar{Y}$ is given as 
$138 \quad \bar{Y}=\frac{4 \omega i l^{2}}{h}\left[\overline{\varepsilon_{33}^{T}}-\frac{2 d_{31}^{2} \overline{Y^{E}}}{(1-v)}+\frac{2 d_{31}^{2} \overline{Y^{E}} Z_{a, e f f}}{(1-v)\left(Z_{s, e f f}+Z_{a, e f f}\right)}\left(\frac{\tan \kappa l}{\kappa l}\right)\right]$

139 where $Z_{a, \text { eff }}$ and $Z_{s, e f f}$ are the mechanical impedances of the PZT and the substrate,

140 respectively; $l$ and $h$ are the half-length and the thickness of the PZT patch, respectively; $v$ is

141 the Poisson's ratio of the piezoelectric material; $\overline{\varepsilon_{33}^{T}}$ is complex dielectric constant; $\overline{\varepsilon_{33}^{T}}=$

$142 \varepsilon_{33}^{T}(1-\delta i) ; \delta=$ dielectric loss factor; $\overline{Y^{E}}$ is the complex modulus of PZT given as $\overline{Y^{E}}=$

$143 Y^{E}(1+i \eta) ; \eta$ is the mechanical loss factor; $d_{31}$ is the piezoelectric strain constant; $k$ is the

144 wave number which is calculated as $k=\omega \sqrt{\frac{\rho\left(1-v^{2}\right)}{Y^{E}}} ; \rho$ is the density of the PZT material; E is

145 the electric field applied for actuation with circular frequency $\omega$ and $i$ is $\sqrt{-1}$.

146 A change in the mechanical impedance of the surrounding medium changes the EI 147 response of the PZT patch. For a concrete substrate, the effectiye dynamic response of the PZT 148 patch was shown to be influenced by the damping and the stress in the substrate [Narayanan 149 and Subramaniam (2016b)]. Considering the high material damping of concrete, the dynamic 150 response of a PZT patch attached to a concrete substrate exhibits a frequency dependence. The 151 vibratory motion of the PZT patch is influenced by the mechanical impedance derived from a 152 zone of finite size. For a PZT patch attached to the concrete substrate, the dynamic response, which consists of distinct modes of vibration of the PZT patch is significantly influenced by 154 the compliance of the material within a zone of influence. The zone of influence for the 155 vibratory motion of a bonded PZT patch depends on the frequency of vibration; the zone of 156 influence is smaller for higher frequencies [Park et al. (2000), Zagrai and Giurgiutiu (2001), 157 Narayanan et al.(2018)]. Any changes in the material compliance within the zone of influence, 158 influences the vibratory motion of the PZT patch. Distributed damage produces an increase in 159 the compliance and the level of damping of the material. Both these effects are known to 
produce a downward shift in frequency and decrease in amplitude of the resonant mode in the EI spectrum.

In this study, an array of surface mounted PZT patches are used for combined EI and AR measurements. As shown in Figure 2, an array of PZT patch sensors is deployed in a structural 164 element, where the EI measurement from the PZT patch is used for local monitoring while the through-transmission measurements are used for distributed sensing. Each PZT patch is used as an actuator and a sensor for through-transmission measurements in the AR mode. A

167 schematic representation of the methodology is given in Figure 2. A received signal undergoes the losses due to signal propagation path and in epoxy at the concrete-beam interfaces.

169 Measures for quantifying changes in the EMI and the received waves due to damage in the 170 form of a localized crack are developed for the level of material discontinuity produced by a stress-induced crack of a given opening.

172

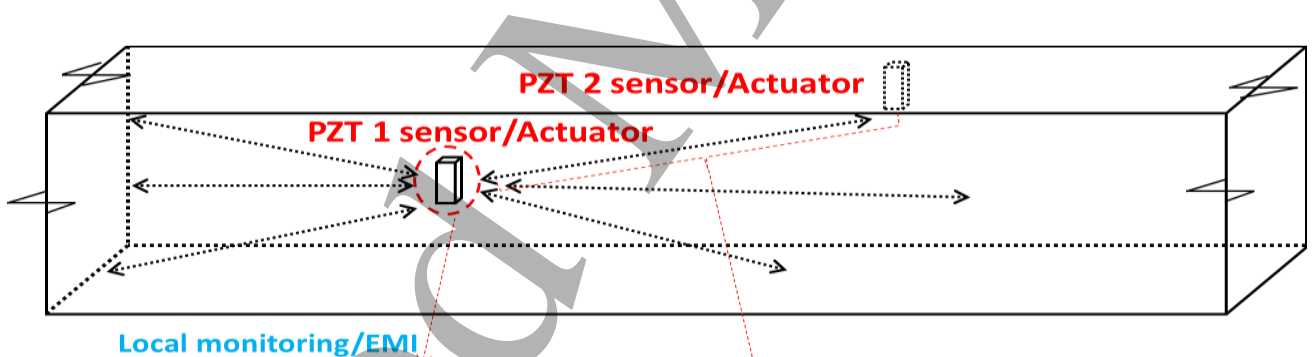

Distributed sensing/Wave propagation

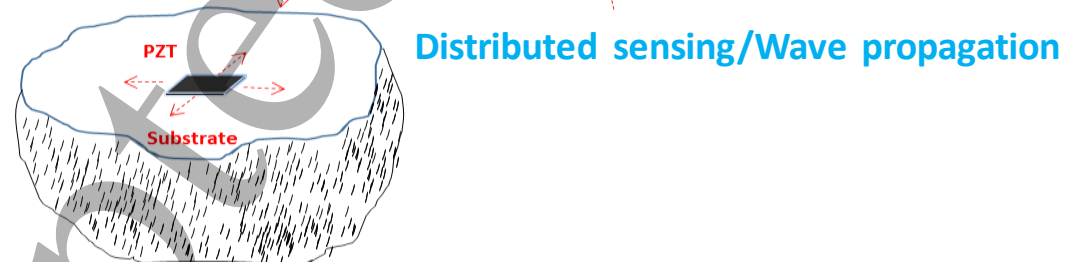

Figure 2. Schematic representation of local-distributed monitoring system using surface mounted PZT patches. 
178

179

180

181

182

183

184

185

186

187

The details of the concrete used in this study is given in Table 1. Coarse aggregate consisted of $20 \mathrm{~mm}$ and $10 \mathrm{~mm}$ crushed gravel in a 1:1 proportion and river sand was used as fine aggregate. Cement conforming to the requirements of OPC grade 53 as per the Indian code of practice, IS 12269:2013 was used. The 28-day compressive strength and modulus of rupture obtained by testing standard $150 \mathrm{~mm}$ cubes and $500 \mathrm{~mm}$ x $150 \mathrm{~mm}$ x $150 \mathrm{~mm}$ sized beams. The properties of the concrete are given in Table 1.

The experimental program consisted of using PZT patches in the distributed (AR) mode and for local EI-based measurements on beams where the damage is induced in the form of a localized crack. A fracture beam was used to produce a crack under flexural loading. The experiments were conducted using notched concrete beams of size $500 \mathrm{~mm}$ (length) x $150 \mathrm{~mm}$ (height) x $150 \mathrm{~mm}$ (width), made with plain cement concrete. A notch, $25 \mathrm{~mm}$ in depth was introduced in the middle and fracture tests were performed using a computercontrolled, servo-hydraulic testing machine The test setup consisted of third point loading as per the requirements of UNI 11039-2:2003 standard. The flexure test was conducted with a span equal to $450 \mathrm{~mm}$ in four-point bending configuration. The fracture test was conducted in crack mouth opening displacement (CMOD) control. The CMOD was increased at rate of $30 \mu \mathrm{m} /$ minute. During the test, the crack tip opening displacement (CTOD) was also measured using a clip gauge mounted at the tip of the notch. Six square $20 \mathrm{~mm}$ PZT patches of $1 \mathrm{~mm}$ thickness were attached to each beam. Properties of the PZT is given in Table 2. Two PZT patches were bonded on front face and two on back face, remaining two were attached to the soffit of the beam. The PZT patches labelled PZT5 and PZT6 were positioned at the bottom of the beam, $50 \mathrm{~mm}$ away from the notch. The other PZT patches were bonded at mid-height of the beam. The PZT patches labelled PZT2 and PZT4 were attached on opposite faces and were located at a distance of $50 \mathrm{~mm}$ from the center of the beam. The PZT patches labelled PZT1 202 and PZT3 were attached on opposite faces of the beam at a distance $50 \mathrm{~mm}$ away from the end 
203

204

205

206

207

208

209

210

211

212

213

214

215

216

217

218

219

220

of the beam. All the PZT patches were bonded to concrete using a two component epoxy. The properties of the epoxy are given in Table 3. The complete test set up showing the locations of the gauges and the PZT sensors is shown in Figure 3a.

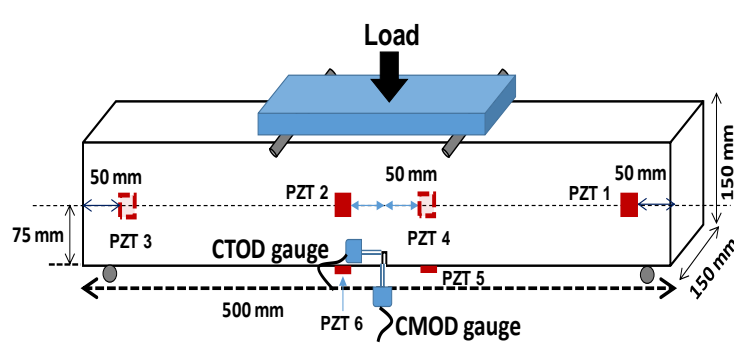

(a)

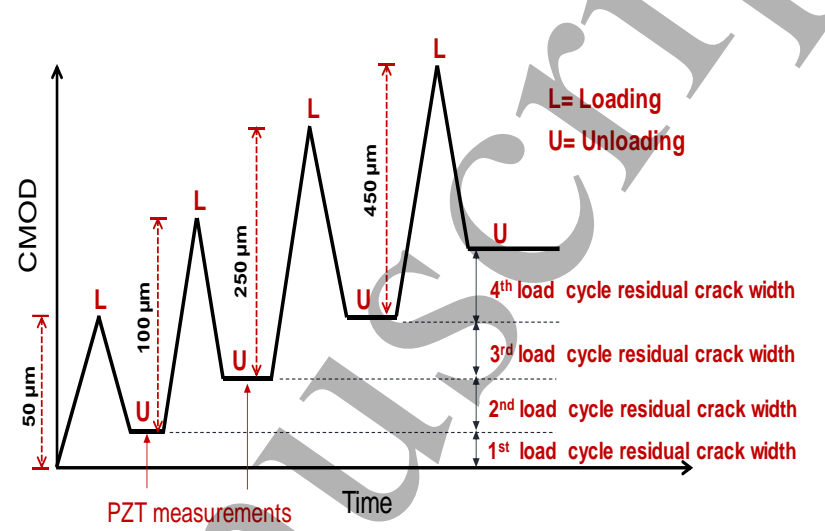

(b)

Figure 3. (a) Test set up for fracture tests with configuration of PZT patches (b) Cyclic loading showing the progressive increase in CMOD.

The test program consisted of cyclic, incremental loading to a larger crack opening in every subsequent cycle; the beam was progressively loaded to predefined values of CMOD. The loading program is shown schematically in Figure 3b. Initially, the beam was loaded up to a CMOD equal to $50 \mu \mathrm{m}$ and unloaded. In the subsequent load cycle, the specimen was loaded to a CMOD value equal to $100 \mu \mathrm{m}$ relative to the CMOD in the unloaded configuration. In subsequent load cycles, the CMOD was increased to values equal to $250 \mu \mathrm{m}$ and $450 \mu \mathrm{m}$ with respect to the residual crack openings at the end of second and third cycles, respectively. In the text, the first, the second, the third and the fourth load cycles are referred to by the maximum relative CMOD values equal to $50 \mu \mathrm{m}, 100 \mu \mathrm{m}, 250 \mu \mathrm{m}$ and $450 \mu \mathrm{m}$, respectively. 
Table 1. Properties of the concrete

\begin{tabular}{|c|c|l|l|l|}
\hline $\begin{array}{l}\text { Mix proportion } \\
\text { (cement: water: fine } \\
\text { aggregate: coarse aggregate) }\end{array}$ & $\begin{array}{l}\text { Density } \\
\left(\mathrm{kg} / \mathrm{m}^{3}\right)\end{array}$ & $\begin{array}{l}\text { Compressive } \\
\text { strength } \\
(\mathrm{MPa})\end{array}$ & $\begin{array}{l}\text { Young's } \\
\text { modulus } \\
(\mathrm{GPa})\end{array}$ & $\begin{array}{l}\text { Modulus of } \\
\text { Rupture } \\
(\mathrm{MPa})\end{array}$ \\
\hline 1:0.45: $1.85: 2.89$ & 2320 & 50 & 33 & 3.6 \\
\hline
\end{tabular}

Table 2. Properties of the PZT material

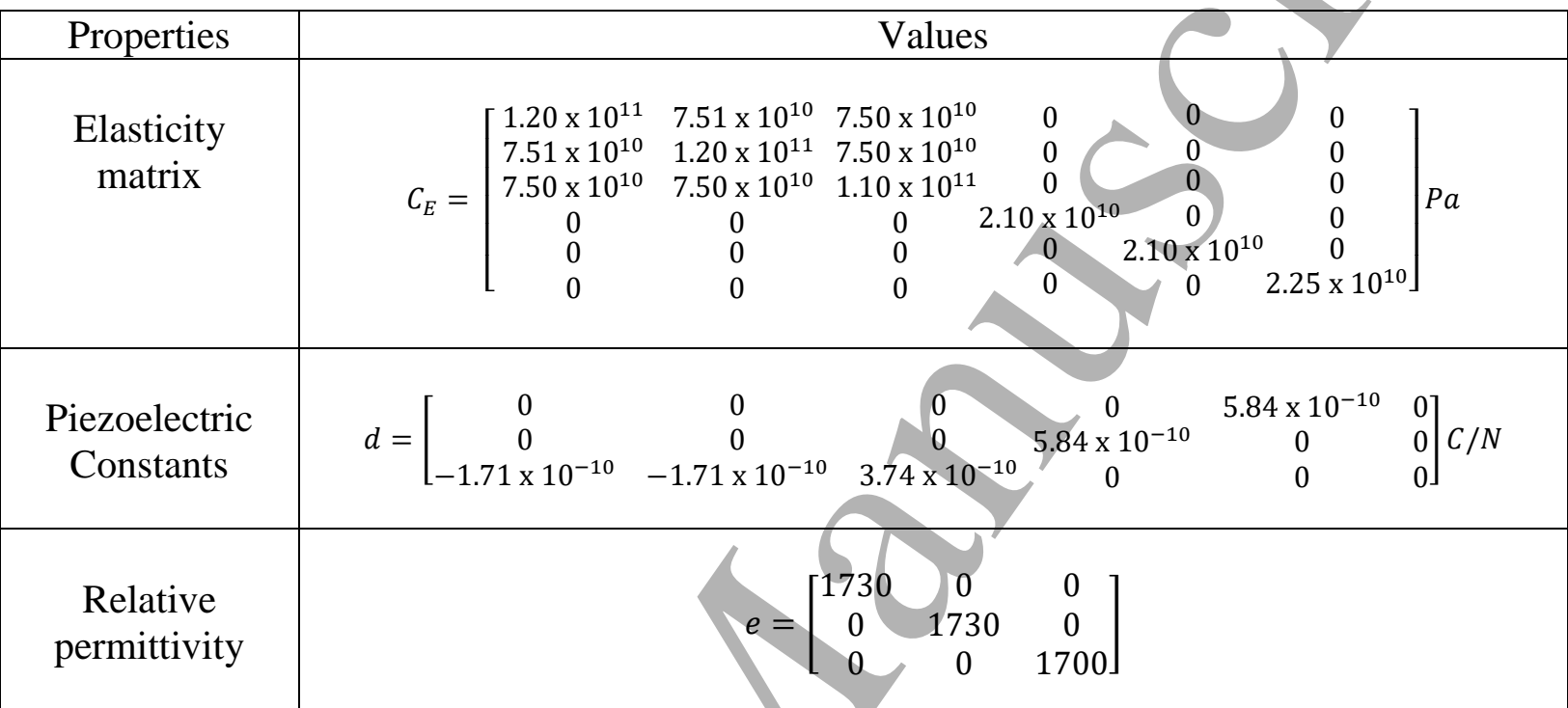

\begin{tabular}{|c|c|c|c|c|}
\hline $\begin{array}{c}\text { Poisson's } \\
\text { ratio, } v\end{array}$ & $\begin{array}{c}\text { Density, } \rho \\
\left(\mathrm{kg} / \mathrm{m}^{3}\right)\end{array}$ & $\begin{array}{c}\text { Dielectric } \\
\text { loss factor, } \delta\end{array}$ & Damping ratio, $\zeta$ & $\begin{array}{c}\text { Mechanical } \\
\text { quality factor, } Q_{m}\end{array}$ \\
\hline 0.35 & 7700 & 0.02 & 0.006 & 75 \\
\hline
\end{tabular}

Table 3. Properties of the Epoxy

\begin{tabular}{|c|c|c|}
\hline Young's modulus (GPa) & Poisson's ratio & Density $\left(\mathrm{kg} / \mathrm{m}^{3}\right)$ \\
\hline 2 & 0.36 & 1300 \\
\hline
\end{tabular}

The EI measurements were performed on all the PZTs before attaching to the beam. The

230 EI measurements were performed at an applied voltage of $1 \mathrm{~V}$ over 800 discrete frequencies 231 ranging between $10 \mathrm{kHz}$ and $500 \mathrm{kHz}$. A 6500B series impedance analyzer of Wayne Kerr 232 make was used for the electrical measurements. The measurements from the PZT patches after 233 attaching to the concrete beam consisted of EI measurements from the individual PZT patches 
234 and through transmission measurements from pairs of PZT patches in the AR mode. A

235 schematic sketch of the test setup used for measurements from the PZT patches is shown in

236 Figure 4. The impedance and the wave propagation measurements were taken after unloading

237 from the predetermined value of CMOD. The experimental set up for the EI and the AR

238 measurements consisted of an impedance analyzer, a function generator, an amplifier, a digital

239 storage oscilloscope and a computer. In the AR mode, the waveform generated by the function

240 generator was amplified and sent to the actuator PZT. Then, the response signal of the

241 remaining five PZTs were logged by the computer, which was interfaced with the digital

242 storage oscilloscope. After acquiring the wave propagation data from all the PZTs, the actuator

243 PZT was switched to the impedance analyzer through the switching unit and the EI

244 measurements were recorded. A typical conductance (real part of admittance) signature of the

245 a PZT patch bonded to beam is shown in Figure 4. This measurement procedure was followed

246 when the specimen is in unloaded state after each load cycle (unloading from CMOD openings

247 equal to $50 \mu \mathrm{m}, 100 \mu \mathrm{m}, 250 \mu \mathrm{m}$ and $450 \mu \mathrm{m}$, relative to the previous unloaded configuration). 


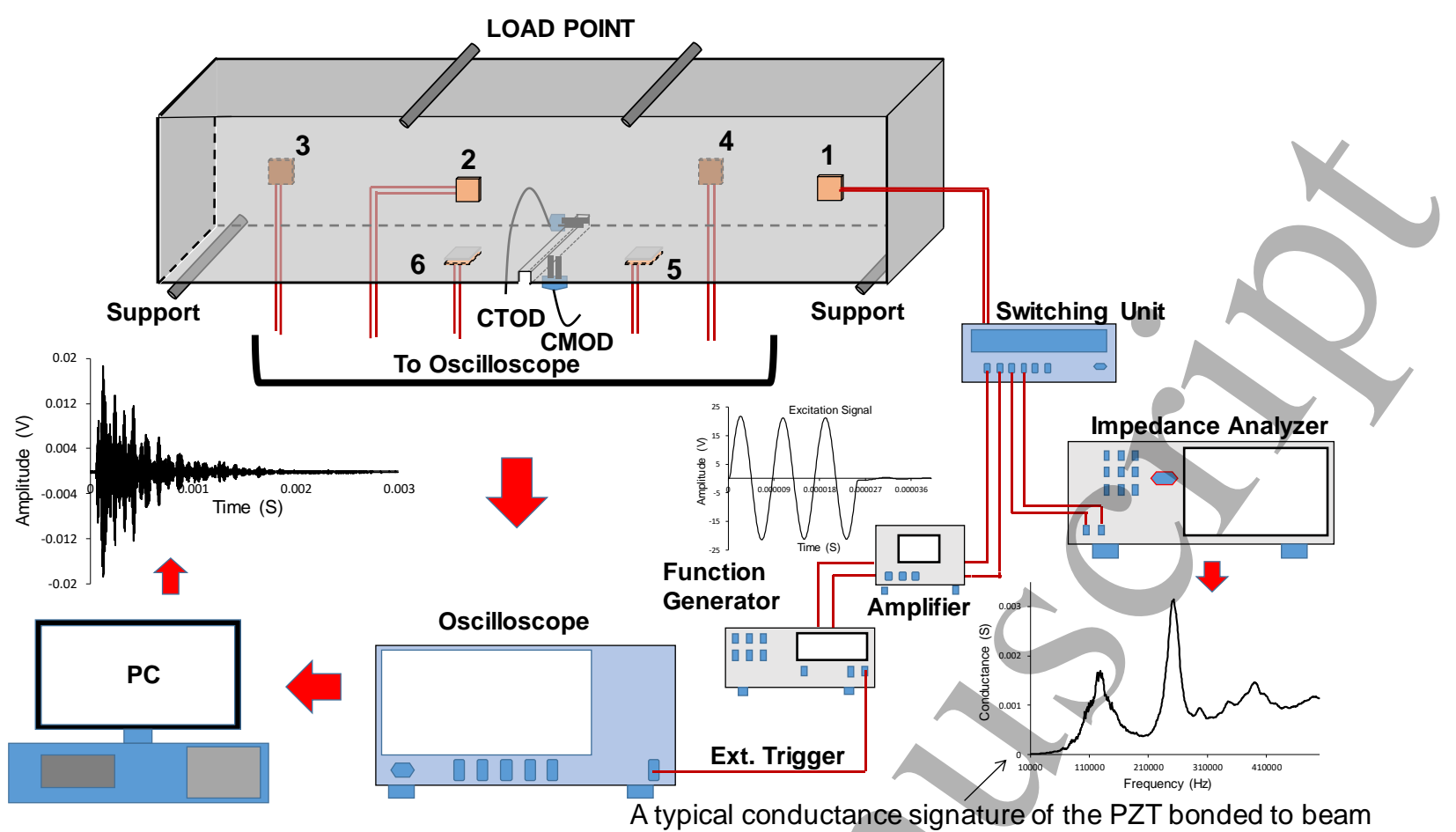

Figure 4. Experimental setups for EI measurements from individual PZT patches and through transmission measurements from pairs of PZT patches in the AR mode.

In the AR mode, the excitation applied to the PZT patches consisted of a 3-cycle tone burst sine signal with center frequency of $120 \mathrm{kHz}$ with a $45 \mathrm{~V}$ peak-to-peak voltage at a pulse repeating frequency equal to $100 \mathrm{~Hz}$ (Figure 5a). The center frequency of $120 \mathrm{kHz}$ was selected to match with the center frequency of the first resonance peak of the bonded PZT to provide higher input and higher sensitivity to received wave. The first resonance peak of the bonded PZT occurs at a low frequency and has the highest energy when compared to other resonance frequencies, which results in lower attenuation of the transmitted waves and a larger received signal. A typical received signal at PZT2 when PZT 1 was actuated $\left(A_{1} R_{2}\right)$ is shown in Figure 5b. All the six PZT patches were individually excited and the response from the all other PZT patches were collected at a sampling frequency of $6.25 \mathrm{MHz}$. 
264

265

266

267

268

269

270

271

272

273

274

275

276

277

278

279

280

281

282 loading. Images of the specimen were captured during the loading program. Each image was 283

Figure 5. Signals from a through-transmission measurement in the AR mode: (a) The excitation signal applied to the actuator (b) The received signal at PZT2 when actuating $\operatorname{PZT} 1\left(\mathrm{~A}_{1} \mathrm{R}_{2}\right)$

During the loading cycles, full-field surface displacements from the beam were obtained using digital image correlation (DIC). DIC measurements were performed on notched specimens to monitor the localization of damage and the subsequent propagation of a crack. DIC relies on correlation between images of specimens in the deformed and the reference unstressed state. A spray painted speckle pattern was created on the surface of the beam. The front face of the beam was initially painted white to create a uniform background. A random pattern was created with a mist of black paint, which was sprayed on the white background. After seating the specimen in the loading setup, the front face of the specimen was uniformly lit with white light. During the flexure test, digital images of the specimen were captured using a camera which was fitted with a $50 \mathrm{~mm}$ lens and was placed at a distance of $1 \mathrm{~m}$ from the specimen surface. A schematic representation of the DIC setup is shown in Figure 6. A digital image of the specimen, referred to as the reference image was captured prior to initiation of

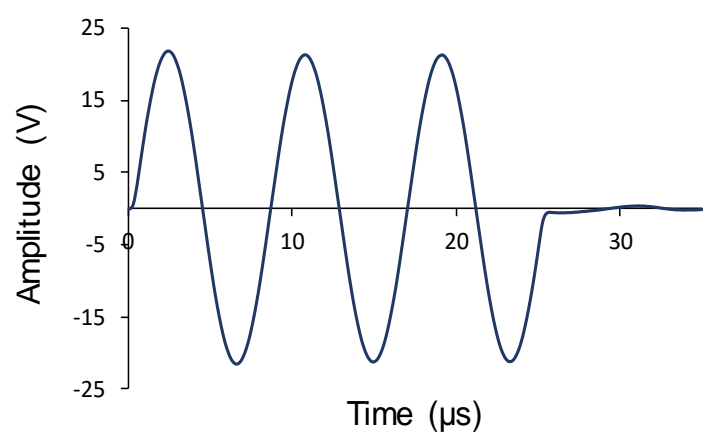

(a)

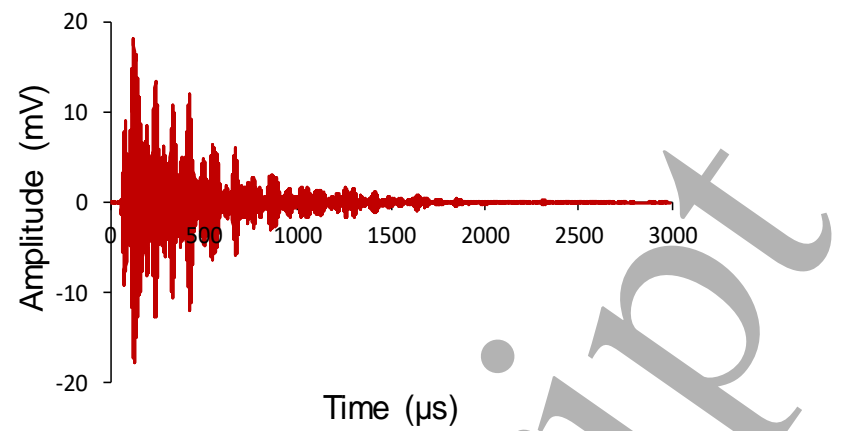

(b) 


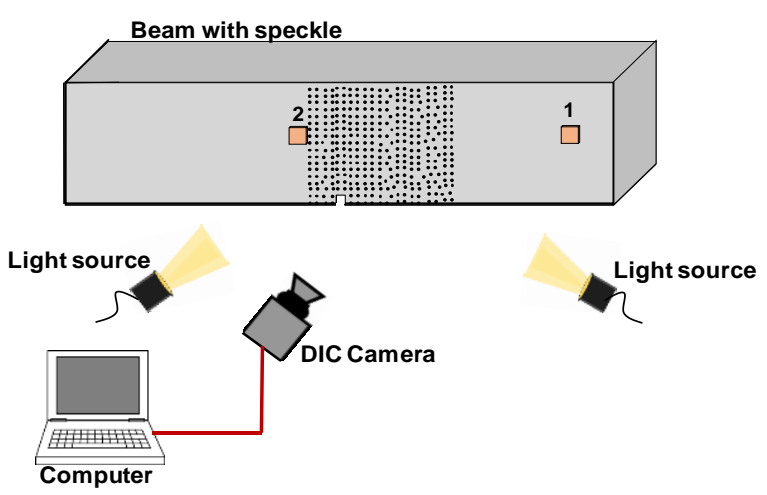

Figure 6. Schematic representation of the DIC setup.

Spatially continuous surface displacements are obtained from correlation between of the random speckle pattern in the deformed and the reference images of the specimen. Analysis for correlations and pattern identification was performed within small neighborhoods called subsets [Bruck et al. (1989), Schreier and Sutton (2002)]. Within each subset, a unique pattern of grey level distribution is formed because of the random sprayed-on pattern. The grey-level pattern in each subset differs from other subsets. The correspondence between matching subsets in images of the specimen in the reference and the deformed states was established using spatial domain cross-correlation. The mapping of positions within the reference image to positions in the deformed images was performed using second-order, two-dimensional shape 298 functions. Sub-pixel level accuracy was obtained using the Quintic B-spline interpolation of 299 the gray values. The cross correlation analysis of the digital images was performed using the 300 VIC-2D ${ }^{\mathrm{TM}}$ software. Strains were calculated from the displacement gradients at each loading stage, by evaluating the shape functions and their partial derivatives at the center of the subset.

302 In the analysis, a subset of size 29 pixels x 29 pixels was used. For the setup used in this study, 303 the random error in the measured displacement was in the range of 0.002 pixels and the resolution accuracy of strain was determined to be $1 \mu \mathrm{m}$. 


\section{Experimental Results}

306

307

308

309

310

311

312

313

314

315

316

317

318

319

320

321

322

323

The load-CMOD responses of three beam specimens are shown in Figure 7a and the loadCMOD response of one beam is shown in Figure $\mathbf{7 b}$ for clarity. The beam was tested in four stages. In each stage, the beam was loaded in CMOD control to a predetermined crack opening relative to the beginning of load cycle and then unloaded to zero load. The EI and wave propagation measurements were taken in the unloaded configuration. The quasi-static load response can be readily identified with the load envelope obtained from the load cycles. The peak load of the envelope load response is attained in the first load cycle. CMOD equal to $50 \mu \mathrm{m}$ is in the post-peak part of the softening load response. There is a continuous increase in the residual CMOD on unloading after each loading cycle. There is also correspondingly a decrease in the stiffness of the load-CMOD response. With every subsequent load cycle, the peak load attained is also smaller.

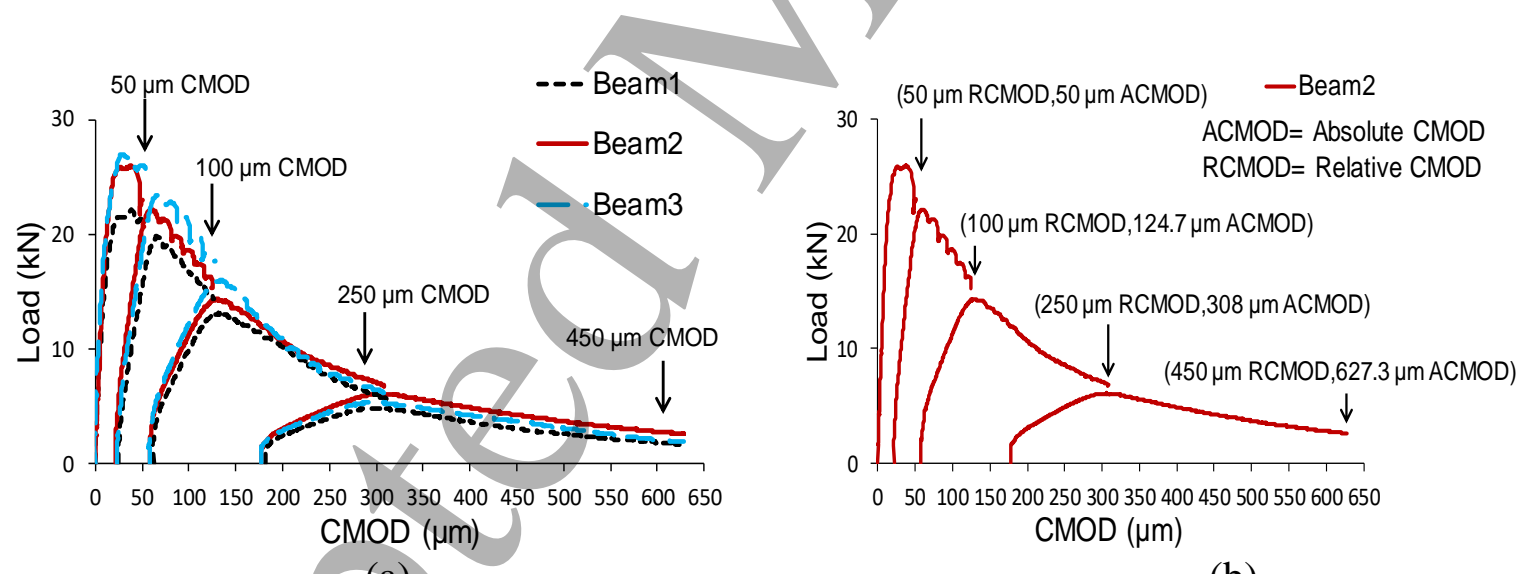

(a)

(b)

Figure 7. (a) Load-CMOD responses of beams; (b) Load-CMOD of Specimen Beam 2. The CMOD relative to the residual CMOD for each cycle are shown. The absolute value of CMOD at the end of each cycle is also indicated in the bracket. 
324 Contours of horizontal strain $\left(\varepsilon_{\mathrm{xx}}\right)$ from the beam specimen 2 at different CMOD values 325 obtained using DIC are plotted in Figure 8. The contours of strain are plotted at the top of the 326 load cycle, just prior to unloading. Localization of the strain can be identified in all the contour 327 plots. The location of the crack can be clearly identified even at a small CMOD equal to $50 \mu \mathrm{m}$.

328 The localized zone propagates along the depth of the beam with increasing CMOD. While the 329 crack could be identified in the contours of $\varepsilon_{\mathrm{xx}}$ even at a small CMOD of $50 \mu \mathrm{m}$, the crack 330 could not be delineated visually up to a CMOD of $124 \mu \mathrm{m}$. There is a sharp gradient in strain 331 within a small region centered on the crack. In the region in the immediate vicinity of the PZT 332 patch, the magnitude of strain is very small and there is no visible damage which produces 333 variation in the measured strain. There is also no visible damage in the form of micro cracking 334 in the region away from the crack.

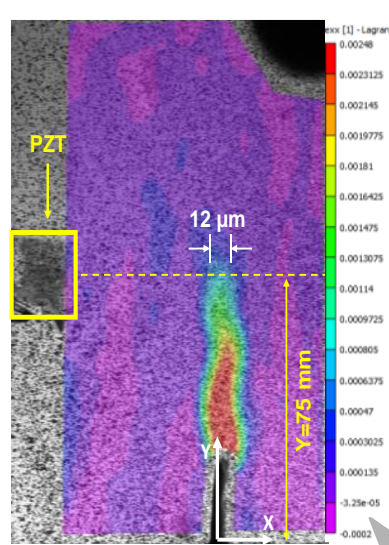

(a)

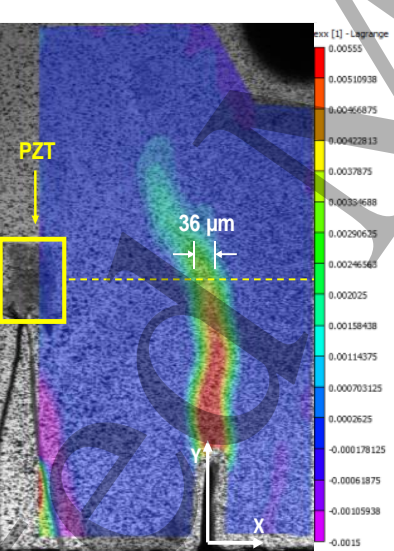

(b)

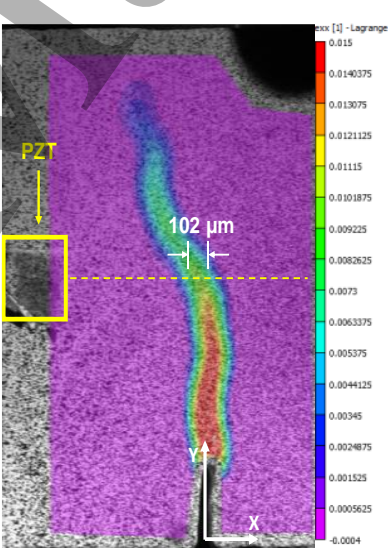

(c)

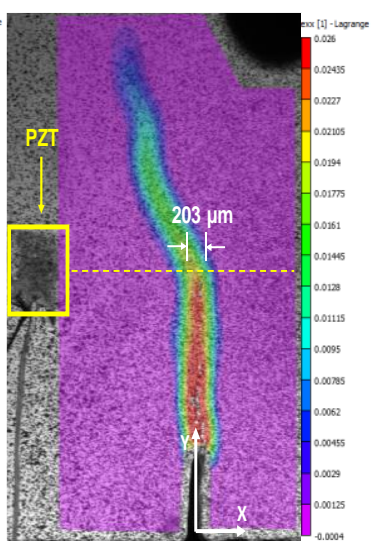

(d)

Figure 8. Strain contour $\left(\boldsymbol{\varepsilon}_{\boldsymbol{x} x}\right)$ at different CMOD levels (a) $50 \mu \mathrm{m}$ (b) $100 \mu \mathrm{m}$ relative to unloading after first load cycle (up to an absolute CMOD equal to $124.7 \mu \mathrm{m}$ ) (d) $250 \mu \mathrm{m}$ relative to unloading after second load cycle (up to an absolute CMOD equal to $308 \mu \mathrm{m}$ ) (e) $450 \mu \mathrm{m}$ relative to unloading after third load cycle (up to an absolute CMOD equal to 
The horizontal strain $\left(\varepsilon_{\mathrm{xx}}\right)$ along length of the beam at the mid-height location along a line with $\mathrm{Y}$ coordinate fixed at $75 \mathrm{~mm}$ above the bottom face and $50 \mathrm{~mm}$ above the notch for various CMOD levels are shown in Figure 9. The variation of $\varepsilon_{\mathrm{xx}}$ along the length shows a sharp increase in the magnitude of strain indicating localization of strain within a small region centered on the notch. It is observed that the width of the localization remains relatively constant with increasing crack opening. With increasing crack opening at the soffit of the beam given by the CMOD, there is an increase in the magnitude of maximum strain. There is also correspondingly a sharper strain gradient within the region of localization with increasing CMOD.

3

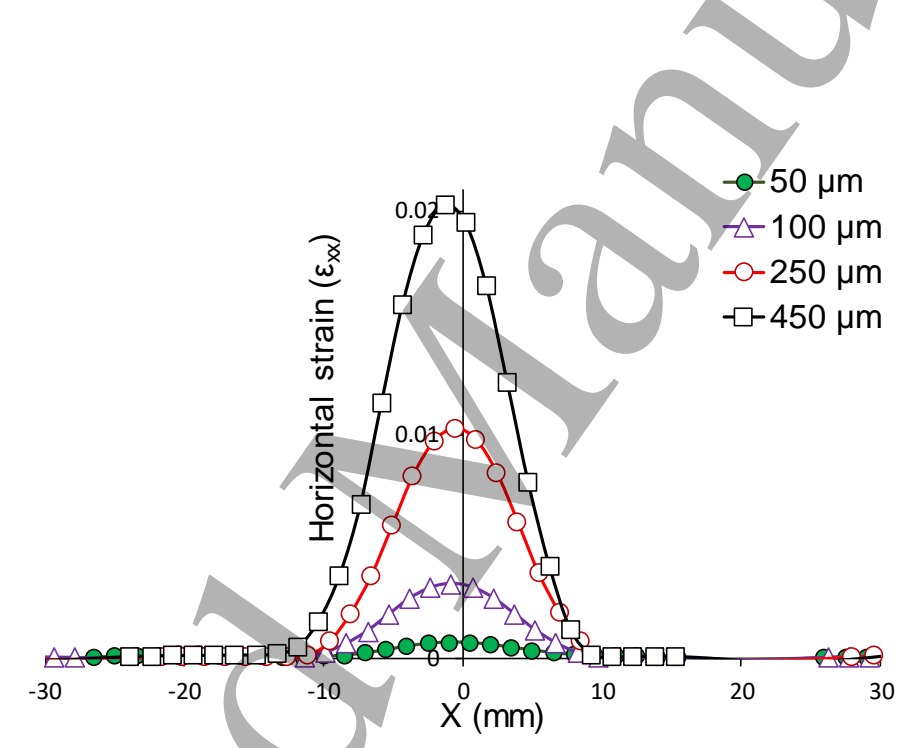

Figure 9. Variation in the horizontal strain $\left(\varepsilon_{\mathrm{xx}}\right)$ with $\mathrm{X}$ coordinate for a line located at $\mathrm{Y}=75 \mathrm{~mm}$ from the bottom of the beam at different values of relative CMOD after each unloading.

The 3D contours of horizontal displacement $\left(\mathrm{U}_{\mathrm{x}}\right)$ obtained at the end of the last load cycle when the CMOD was increased to $450 \mu \mathrm{m}$ from the unloaded configuration, is shown in Figure 10a. The crack in the medium is identified by the displacement discontinuity emanating from the notch. The profile of the crack is identified by the sudden increase in the $U_{x}$ over a 
small region. The discontinuity in $U_{\mathrm{x}}$ introduced by the crack emanating from the notch is evident in the jump in the $\mathrm{U}_{\mathrm{x}}$. The presence of the crack and the associated physical opening within a subset resulted in a loss of correlation within the subsets in which the crack was present. The loss of correlation and the finite size of the pixel resulted in smearing of the displacement gradient within a region of size equal to the subset size close to the crack. The actual width of the region of localization produced by the crack is therefore smaller than the width of the zone with high strains indicated in Figure 9. A procedure for obtaining the crack opening precisely, free from the error introduced by the finite subset size was developed using the asymptote matching procedure [Reddy and Subramaniam (2017), Gali and Subramaniam (2018)]. The crack opening widths along the depth of the beam at different values of CMOD obtained using the asymptote matching procedure and are shown in Figure 10b for beam specimen 2. The CMOD corresponds to the displacement measured across the notch at $\mathrm{Y}=0$, using the CMOD gauge located at the bottom of the beam. The CMOD is also shown marked in the figure at the location corresponding to $Y=0$. The crack opening widths were determined from the $\mathrm{U}_{\mathrm{x}}$ measured using DIC. Corresponding to the CMOD measured on the soffit of the beam, there is a decrease in the crack opening width with increasing Y coordinate above the notch. The zero crack opening along the depth of the beam gives the physical location of the tip of the propagating crack. The observed crack opening as a function of depth for different values of CMOD indicates that the crack tip progresses along the depth of the beam with increasing CMOD. For a CMOD of $50 \mu \mathrm{m}$, the crack has propagated along the depth and the tip of the crack can be identified at $86 \mathrm{~mm}$ from bottom of the beam and the corresponding crack opening at the mid-height location is $12 \mu \mathrm{m}$. At a CMOD equal to $100 \mu \mathrm{m}$ relative to unloaded configuration at the end of the first load cycle (absolute CMOD equal to $124.7 \mu \mathrm{m}$ ), the tip of the crack is located at $\mathrm{Y}=119 \mathrm{~mm}$ and the crack opening at the mid-height location

387 is $36 \mu \mathrm{m}$. In the subsequent load cycles when the CMOD is increased to $250 \mu \mathrm{m}$ and $450 \mu \mathrm{m}$ 


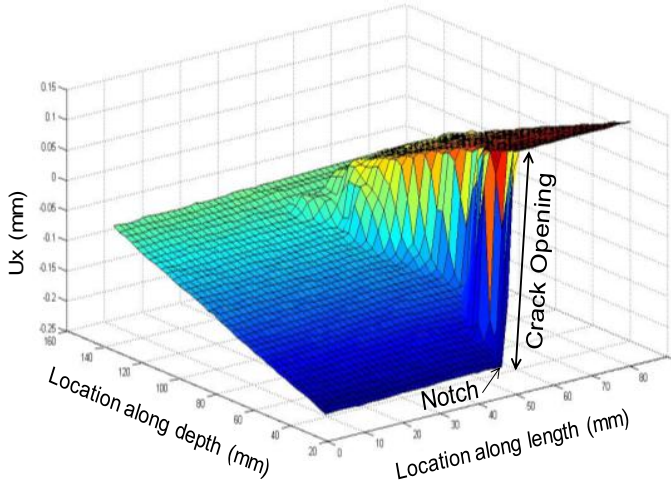

(a)

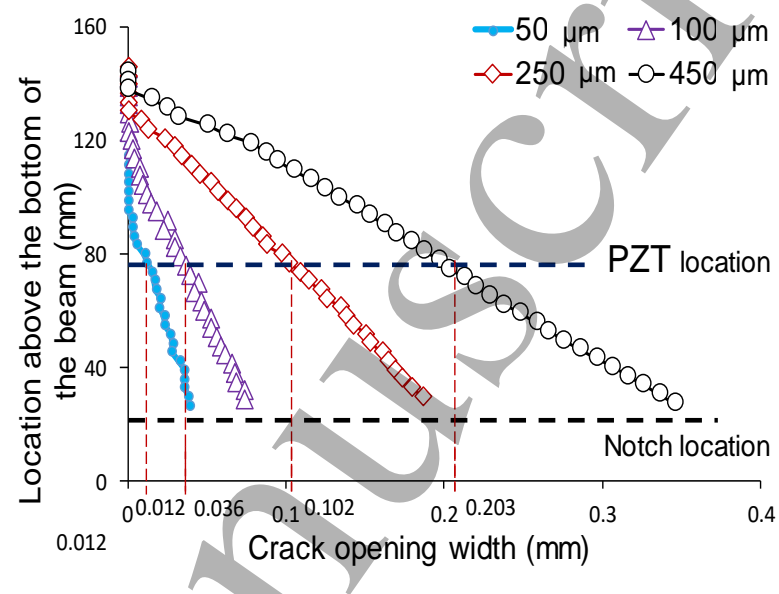

(b)

Figure 10. (a) Contour showing crack opening displacement at the relative value of CMOD $=450 \mu \mathrm{m}$ after the third cycle; (b) Crack opening width as a function of depth of the beam at different crack mouth opening displacements.

\subsection{Measurements from PZT patches}

The stress wave attenuation measurements were performed in the unloaded state, where the removal of the load results in closing of the crack. The measured changes recorded by the PZT patches therefore correspond to the physical discontinuity in the medium produced by the stress-induced crack. Each PZT patch was individually actuated (Figure 11b) and the responses were recorded at all the other PZT patches. This measurement procedure was repeated when the specimen was in the unloaded state after pre-determined crack opening indicated by different relative values of CMOD $(50 \mu \mathrm{m}, 100 \mu \mathrm{m}, 250 \mu \mathrm{m}$ and $450 \mu \mathrm{m})$. The

406 level of noise obtained from the standard deviation of the initial part of the signal was on the 
407

408

409

410

411

412

413

414

415

416

417

418

419

420

421

422

423

424

order of $0.1 \mathrm{mV}$. All the received signals were conditioned with a linear phase, band-pass filter.

A comparison of actual received signal and filtered received signal is shown in Figure 11a.

Figure 11b shows a typical signal received by PZT2 using PZT1 as the actuator $\left(A_{1} R_{2}\right)$ at the seating load, prior to initiation of the first cycle of loading. The received signal is significantly smaller in magnitude when compared with the excitation. The decrease in the magnitude of the received signal is attributed to losses in the path of the wave produced by the geometric spreading of the wave and the material attenuation in the wave path in the epoxy and in the concrete. There is also a significant increase in the length of the received wave when compared with the excitation applied, which is due to several effects such as, the ringing of the PZT patch, geometric spreading and multiple reflections, mode conversions and from inhomogeneity of the beam specimen [Aggelis and Shiotani (2007)].

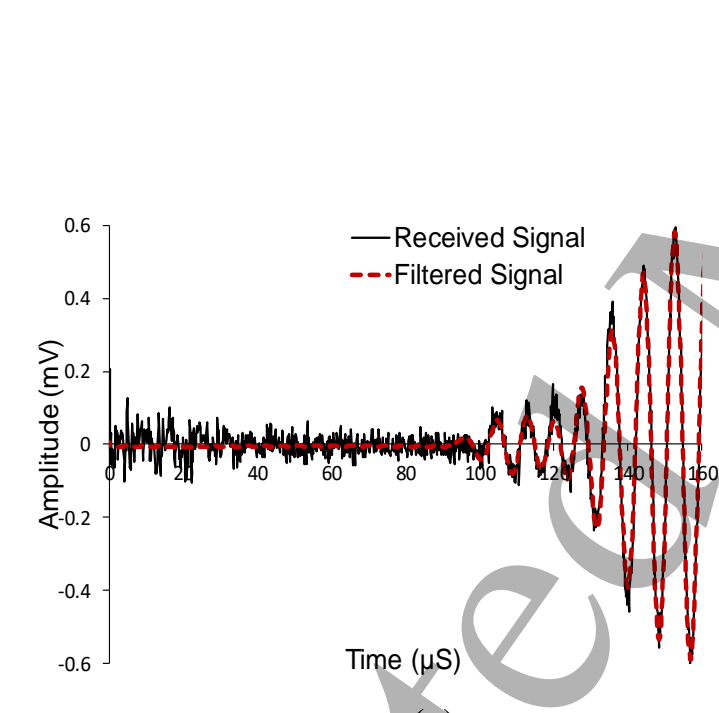

(a)

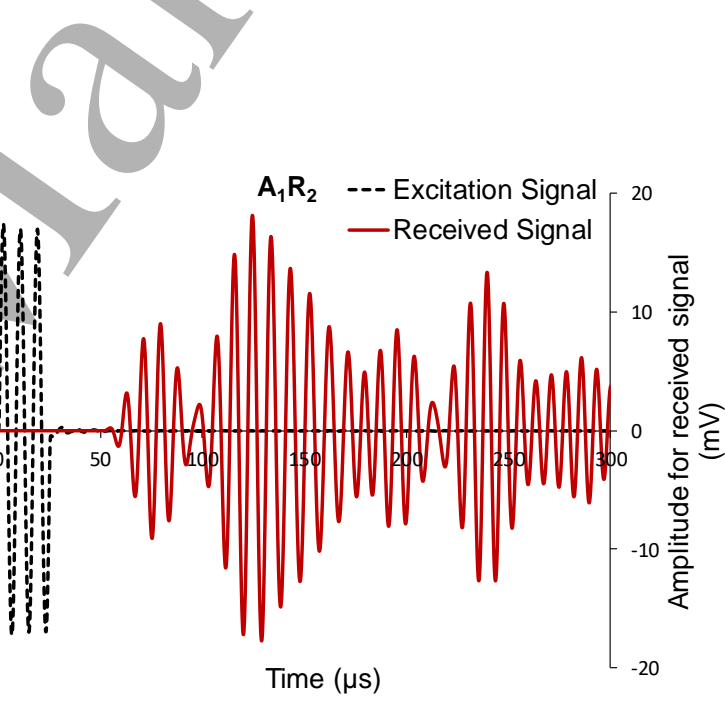

(b)

Figure 11. (a) Comparison of the actual received and the filtered received signal (b)

Comparison of the actuating signal with sensor signal prior to loading and the received signal after filtering. 
Figure 12 shows the signals recorded from different PZT sensors (PZT2, PZT4, PZT6)
426

427

428

429

430

431

432

433

434

435

436

437

438

439

440

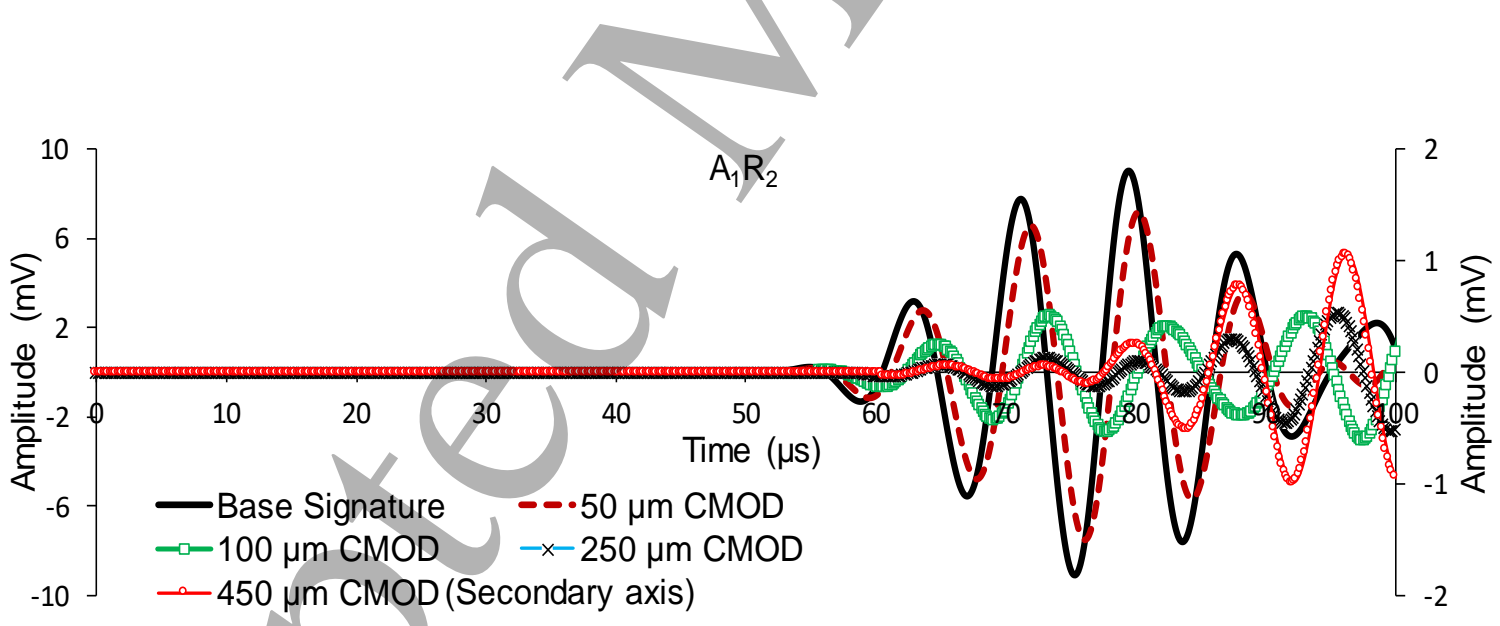
when PZT1 was actuated. The received signals at PZT6 have a smaller amplitude when compared with the signals recorded by PZT2 and by PZT4 even in the pristine stage. The amplitude of the signal is influenced by the material and geometric attenuation in path of the transmission. Each subfigure in Figure 12 shows the variation in the received signal in the unloaded state after each cycle. The changes recorded at the different PZT/patches however vary depending on the positions of the PZT patches relative to the notch. With increasing CMOD, there was a decrease in the amplitude and an increase in the time of arrival of the stress waves received at PZT2 and PZT6 when compared with the corresponding baseline signatures. The changes in the received waves are produced by the presence of the crack in the path of stress wave propagation. The received signals of PZT4 are not significantly altered since the wave path does not intersect the crack. The received signals at PZT4 exhibit a small change which may be attributed to stress wave reflection from the crack surface.

441 
442

443

444

445

446

447

448

449

450

451

452 after each load cycle with base line signature, the signals received at PZT 2 and PZT6 undergo

453

454 also show that there was $\varepsilon_{\mathrm{xx}}$ concentration beyond the mid height of the beam in the $\mathrm{A}_{1} \mathrm{R}_{2}$

455 transmission path for all four CMOD levels. There is an increasing level of material 456 discontinuity in the propagation path produced by the crack after each load cycle.

(b)

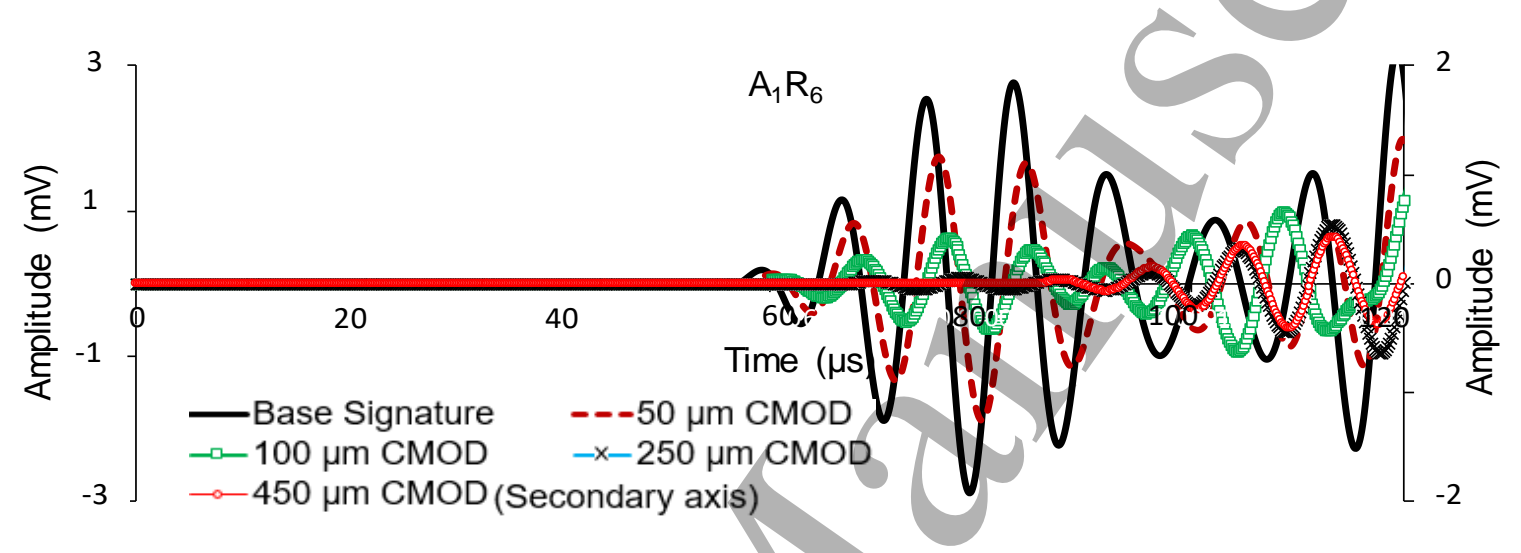

(c)

Figure 12. The received signals when PZT1 is actuated for different crack opening displacements: (a) received at PZT2 (b) received at PZT4 (c) received at PZT6.

When PZT1 is actuated, the stress wave signals received at the other PZT patches depend on the stress wave transmission path. Any changes in the received signal characteristics are produced by changes in the stress wave transmission path. When comparing the sensor signals changes in the time of arrival and the energy content. The contours of $\varepsilon_{\mathrm{xx}}$ shown in Figure 9

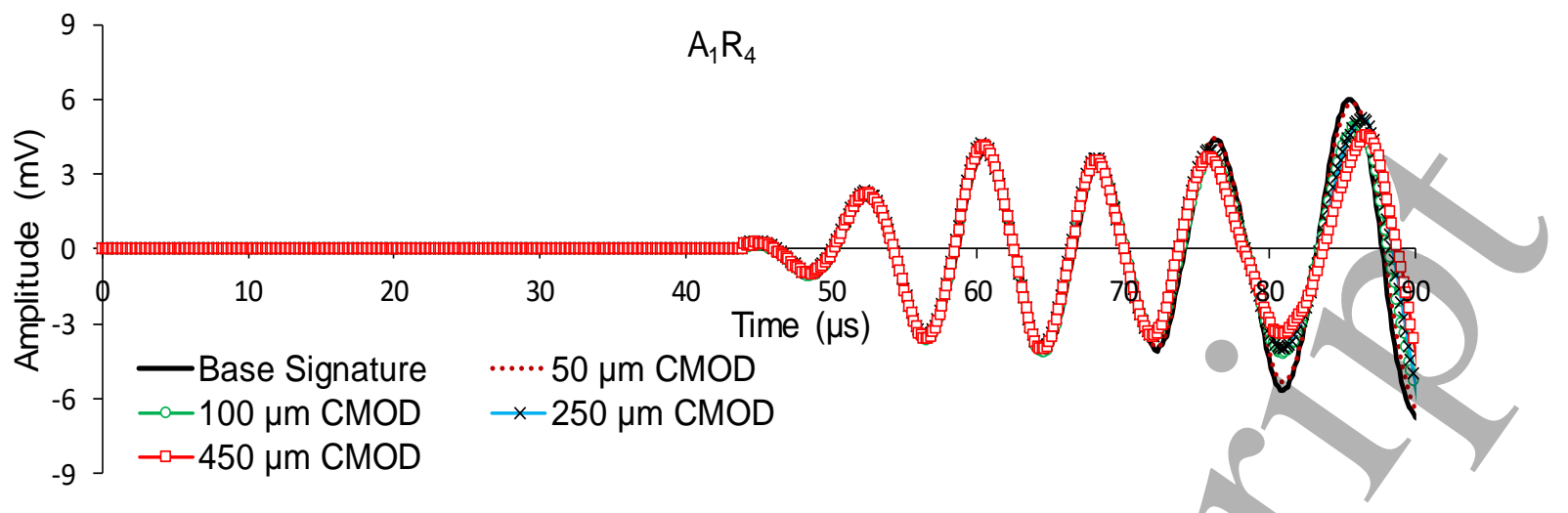


457 Correspondingly, there is a larger decrease in the amplitude and a larger increase in the arrival 458 time in the received signals. After the first load cycle, the crack was not visually apparent and 459 was only detected from the displacement discontinuity recorded using DIC. From the crack 460 opening width as a function of height shown in Figure 10b, for a CMOD equal to $50 \mu \mathrm{m}$, the 461 crack propagating depth was $86 \mathrm{~mm}$ from bottom of the beam and the crack opening 462 displacement at the mid-height in the loaded configuration was $12 \mu \mathrm{m}$. The crack propagated 463 beyond the direct wave path of $\mathrm{A}_{1} \mathrm{R}_{2}$ (actuating PZT1 and receiving PZT2) even at the CMOD 464 equal to $50 \mu \mathrm{m}$.

465 Typical electrical conductance spectra recorded from EI measurements from the six PZT 466 patches attached at different locations on the beam specimen 2 are shown in Figure 13. Distinct 467 resonance modes are clearly identified with peaks in the spectra. The relative locations and 468 amplitudes of the peaks in the conductance spectra are relatively constant. The variations in 469 the absolute values of amplitudes and the center frequencies of the individual peaks are due to 470 variations in the properties of the individual PZT patches and the thickness of the epoxy used 471 for bonding the PZT patch to the concrete substrate. The first and the second resonance peaks 472 are centered on $120 \mathrm{kHz}$ and $250 \mathrm{kHz}$, respectively. The peaks of resonance have previously 473 been shown to be sensitive to changes in substrate compliance within their respective zones of 474 influence [Narayanan and Subramaniam (2016a, b)]. The zones of influence for the first and 475 the second resonant peaks for $1 \mathrm{~mm}$ thick, $20 \mathrm{~mm}$ square patches have been shown to be $476150 \mathrm{~mm}$ and $100 \mathrm{~mm}$, respectively. The local peaks on the first resonant peak are identified 477 with the influence of the boundary of the specimen, which is within its zone of influence 478 [Narayanan et al. (2018)]. The presence of distributed damage, which influences the 479 mechanical impedance of the substrate in the zone of influence has been shown to produce 480 changes in the amplitude and center frequency of the resonant peak. 


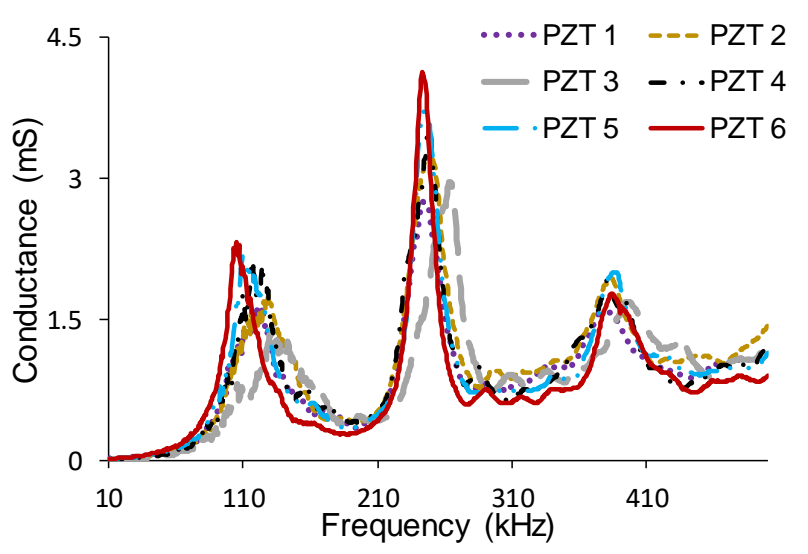

Figure 13. The electrical conductance spectra recorded from the PZT patches

The EI measurements were recorded from the PZT patches in the unloaded state. The conductance spectra over frequencies centered on the first and second peaks for PZT2 and PZT5 (50mm away from notch) and PZT3 (180mm away from notch) are shown in Figure 14.

The electrical conductance obtained from the PZTs centered on the first resonant peak are shown in Figures 14 a, b, c. A change is noticed in the EI response of all the PZTs. As the crack is located away from the zone of influence of first peak for PZT 3, the changes are minimal. For PZT 2 and PZT5, there are irregular changes identified in the local peaks due to 492 the presence of the material discontinuity within the zone of influence of the first peak of the 493 PZTs.

The electrical conductance obtained from the PZTs centered on the second peak are shown in Figures 14 d, e,f. There were no changes in the second peak of EM response of PZT 3 since the crack lies outside its zone of influence [Narayanan et al. (2018]. There are small changes 497 in conductance signatures centered on the second resonant peak for PZT2 and PZT5 at the 498 different levels of CMOD as the crack passes through the zones of influence. The 499 measurements from DIC indicated that the crack was very localized while the material away 500 from the localized zone had no damage. Considering no visible distributed damage, the 
501 presence of localized damage within the zone of influence does not appear to significantly

502 influence the mechanical impedance offered by the concrete medium to the motion of the PZT

503 in the second resonant mode. The second resonant mode is therefore not sensitive to the

504 presence of a localized material discontinuity produced by a stress-induced crack in concrete.

505

506

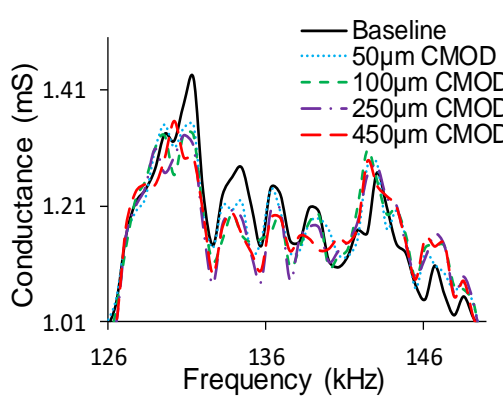

(a)

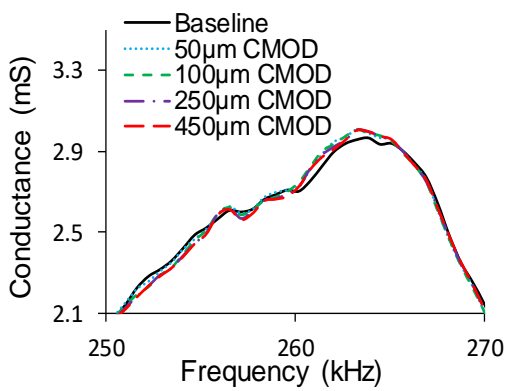

(d)

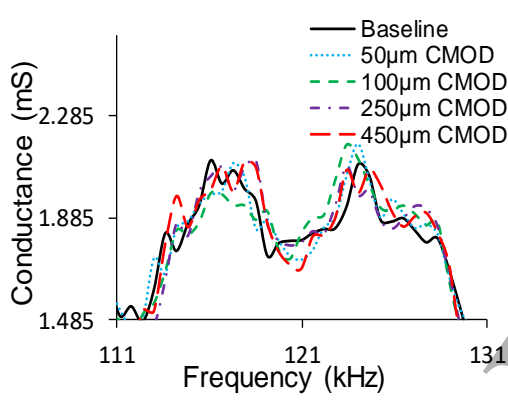

(b)

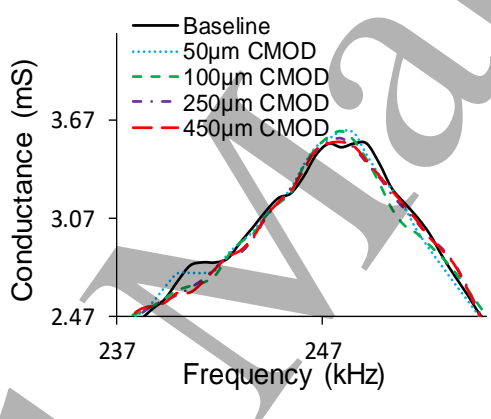

(e)

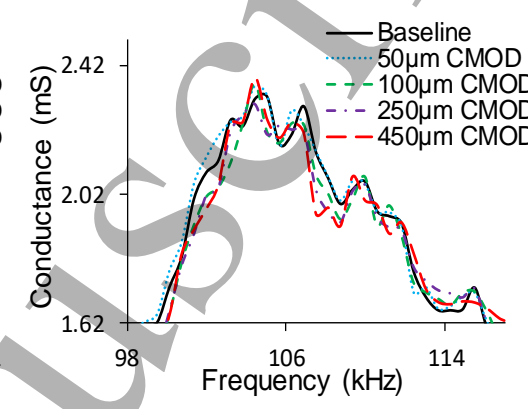

(c)

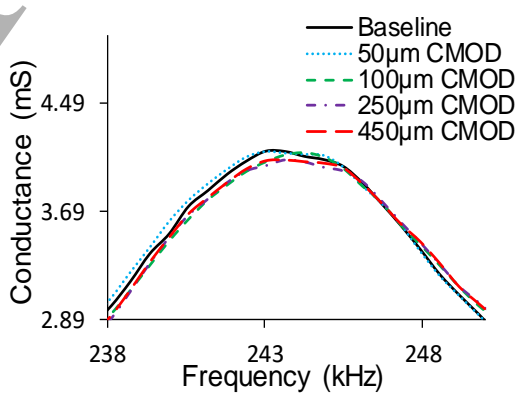

(f)

510 Figure 14. Electrical conductance spectra close to first peak at different CMOD levels of (a)

511 PZT 3 (b) PZT 2 (c) PZT 5. Conductance spectra close to second peak at different CMOD

512 levels of (d) PZT 3 (e) PZT 2 (f) PZT 5.

\section{Analysis of Results}

515 The experimental studies conducted on the beam at different levels of CMOD indicate that

516 there is a consistent change in the recorded parameters induced by the localized crack. The 517 stress-induced crack in concrete is associated with a physical opening under applied loading 
518

519

520

521

522

523

524

525

526

527

528

529

530

531

532

533

534

535

536

537

538

539

540

541

and it produces a discrete discontinuity in the material in the unloaded state. The discrete crack present in the concrete is also very localized and does not produce any additional strain or damage in the bulk of the medium. From the DIC measurements it was established that even at a CMOD equal to $50 \mu \mathrm{m}$, the crack had propagated to a depth of $86 \mathrm{~mm}$ above the notch. The physical opening at the mid-height was determined to be $12 \mu \mathrm{m}$ in the loaded state when the CMOD was $50 \mu \mathrm{m}$. With an increase in the CMOD there was an increase in the physical crack opening along the depth of the crack. The opening displacement produced by a stress induced crack creates a physical discontinuity in the material medium. The measurements from the PZT patches were performed in the unloaded state, and these correspond to the material discontinuity produced by the stress-induced crack.

A quantification of the observed changes is performed to identify the changes in the wave characteristics and the EI measurements produced by the presence of the localized crack in the medium. In the AR measurements, the changes are observed in the time of flight of stress wave arrival and in the amplitude of stress wave. Percentage changes in the time of flight (TOF) and the attenuation factor $(\mathrm{A}(f))$ were used to quantify the changes in the received signal in the AR mode. Changes in the conductance signature at the resonance peaks of the EI response were quantified using the root mean square deviation (RMSD).

The changes in propagation path of stress wave due to material discontinuity produce changes in the time of flight (TOF) of the received signal. The percentage $(\%)$ change in the TOF at each level of CMOD was calculated using Equation (2), where $(T O F)_{d}$ is the TOF at different CMOD and $(T O F)_{0}$ is the TOF at zero CMOD. Time of flight of the signals were calculated using voltage thresholding technique where a threshold value $(5 \mu \mathrm{V})$ was set for the signal and the time at which the received signal crosses threshold is taken as TOF. The threshold voltage was set based on maximum noise level present in the signal. 
542

$\Delta T O F(\%)=\frac{\left[(T O F)_{d}-(T O F)_{0}\right]}{(T O F)_{0}} * 100$

543

544

545

546

547

548

549

550

551

552

553

554

555

556

557

558

559 measured opening.

The $\Delta \mathrm{TOF}(\%)$ for the different AR pairs are plotted as a function of the different stress-induced crack opening at the mid-height of the beam in Figure 15. The physical crack opening at the mid-height location of the beam determined using DIC are used for the plot. The values corresponding to reverse excitation of PZTs are not plotted since these values were nominally identical. After the first cycle of loading, there is an increase in the $\triangle \mathrm{TOF}(\%)$ in the received signals at the different PZTs. In the AR pairs, where the wave path did not intersect the crack, there was no change in the $\Delta \mathrm{TOF}(\%)$. In the subsequent load cycles when the relative value of CMOD was equal to $100 \mu \mathrm{m}, 250 \mu \mathrm{m}$ and $450 \mu \mathrm{m}$ there is a further increase in the $\Delta \mathrm{TOF}(\%)$ for in the AR pairs with signal path crossing the crack $\left(A_{6} R_{5}, A_{1} R_{3}, A_{3} R_{4} . A_{4} R_{6}, A_{3} R_{5}\right.$ and $\mathrm{A}_{1} \mathrm{R}_{6}$ ). The measurements in $\triangle \mathrm{TOF} \%$ progressively increase with an increase in the physical discontinuity in the concrete produced by the stress-induced crack associated with the

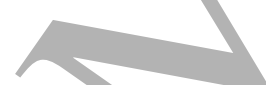

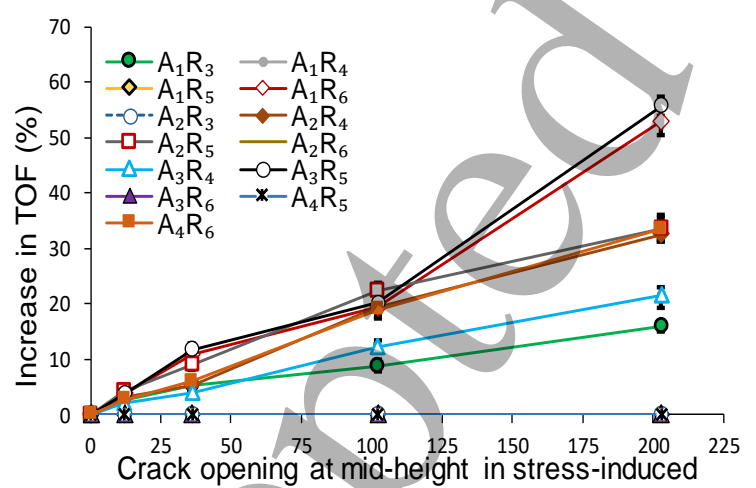

crack $(\mu \mathrm{m})$

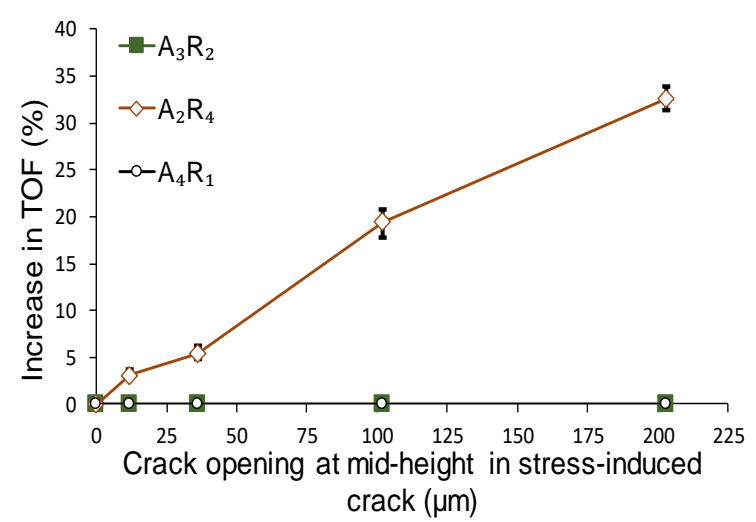

(b)

Figure 15. (a) $\Delta \mathrm{TOF}(\%)$ at different CMOD values. b) $\Delta \mathrm{TOF}(\%)$ for transmission paths $\mathrm{A}_{3} \mathrm{R}_{2}, \mathrm{~A}_{2} \mathrm{R}_{4}$ and $\mathrm{A}_{4} \mathrm{R}_{1}$ 
560 The $\Delta \mathrm{TOF}(\%)$ for transmission paths $\mathrm{A}_{3} \mathrm{R}_{2}-\mathrm{A}_{2} \mathrm{R}_{4}-\mathrm{A}_{4} \mathrm{R}_{1}$, which connect PZTs located at mid-

561 height, are plotted as a function of stress-induced crack opening in the stress wave path in

562 Figure 15b. There is no change on the $\Delta \mathrm{TOF}(\%)$ measured in the signal path $\mathrm{A}_{3} \mathrm{R}_{2}, \mathrm{~A}_{4} \mathrm{R}_{1}$. In

563 the path $\mathrm{A}_{2} \mathrm{R}_{4}$ there is consistent increase in $\triangle \mathrm{TOF}(\%)$ with an increase in the stress induced

564 crack opening. Changes in the time of flight of the received signal are attributed to the presence

565 of the material discontinuity in the stress wave path. The $\Delta \mathrm{TOF}(\%)$ therefore provides a

566 reliable parameter for detecting changes in the transmission of the stress wave due to the

567 discontinuity in the material medium produced by a localized stress-induced crack in concrete.

568 A comparison between the actuated signal and the received signal prior to initiation

569 of loading indicates that there is significant attenuation in the path of wave. When one

570 PZT pair is used in the AR mode it is of interest to determine the additional attenuation

571 produced by the material damage. The influence of attenuation in the stress wave path

572 produced by the materials, concrete and epoxy, have to be separated to determine the

573 additional attenuation produced by the crack. The changes in the received signals due to

574 the propagation of crack depend on the distance between actuator and sensor, depth and

575 width of crack. In an array of sensors where comparison among different transmission

576 paths are necessary to identify the location and the severity of the crack, a new damage

577 index known as Attenuation factor $(A(f))$ is introduced in the analysis for compensating

578 the intervening effects such as properties of PZTs, amplitude of the resonance frequencies

579 of PZTs, epoxy concrete interface losses and length of direct propagation path. The

580 procedure is similar to the self-calibrating technique where the signal processing in the

581 AR mode consisted of normalizing signals in the frequency domain [Achenbach et al.

582 (1992), Wang and Subramaniam (2011)]. The received signal in time domain signal was

583 transformed to the frequency domain using the FFT algorithm. The direct stress

584 transmission in an AR pair is the stress wave in the early part of the received signal. The 
585 signal received subsequently contains reflections from the boundaries and edges. The initial

$58626 \mu$ s (length of actuating signal) signal starting from time of flight of the received signal was

587 taken as the direct stress wave (non-reflected signal) as shown in the Figure 16a. The FFT of

588 non-reflected time domain signal with the corresponding half power bandwidth was shown in

\section{Figure 16b.}

590

591

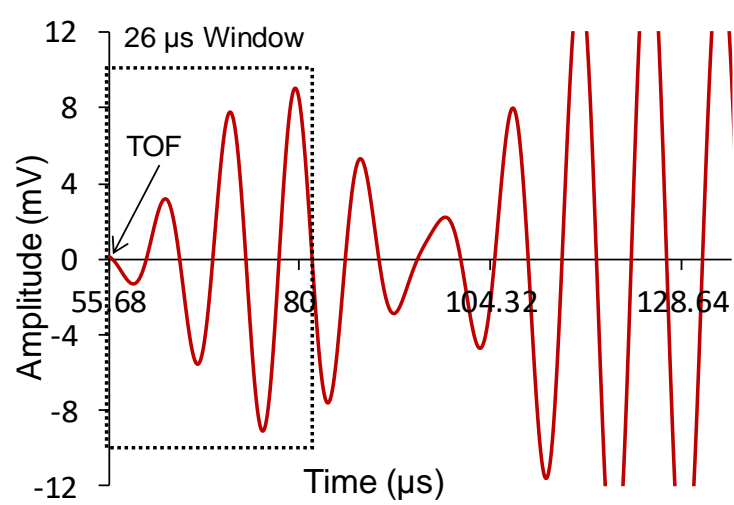

(a)

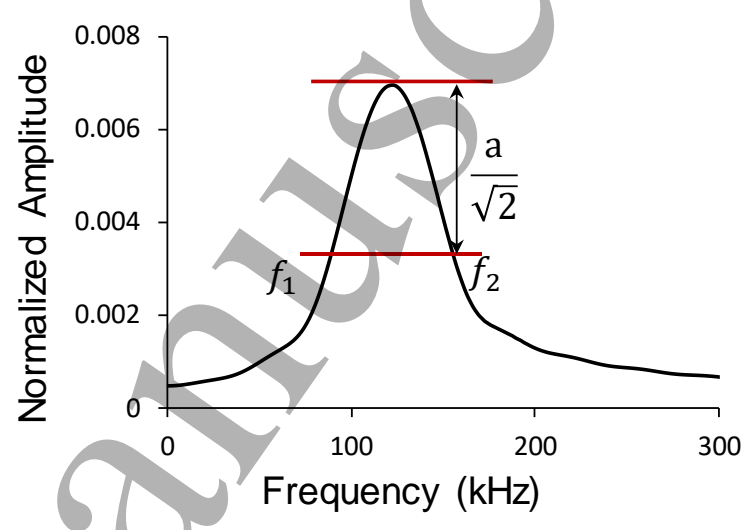

(b)

$593 \quad$ Figure 16. (a) Non-reflected signal $(26 \mu$ s) (b) FFT of the received non-reflected time 594 domain signal $(26 \mu \mathrm{s})$

595

596 The received signal in a through transmission measurement in time domain contains influences 597 of losses due to propagation media and can be expressed as,

598 $r_{s}=$ $a_{s} * l_{e a} * l_{s p} * l_{e s}$

601 where $r_{s}$ is the received signal, $a_{s}$ is the actuating signal, $l_{e a}, l_{e s}$ are the signal losses in the 602 layer of epoxy present at the actuator and sensor, respectively, $l_{s p}$ is the due to propagation 
603 through concrete, and ' $*$ ' is the convolution operator. The received signals at zero CMOD $604\left(r_{s}(f)_{0}\right)$ and at different CMOD levels $\left(r_{s}(f)_{d}\right)$ can be expressed as,

$605 r_{s}(f)_{0}=a_{s}(f) \cdot l_{e a}(f) \cdot l_{s p}(f) \cdot l_{e s}(f$

$606 r_{s}(f)_{d}=a_{s}(f) \cdot l_{e a}(f) \cdot l_{s p}(f) \cdot l_{e s}(f) \cdot L_{d}(f)$

607 where $r_{s}(f)_{0}, r_{s}(f)_{d}$ are the magnitudes of FFT at zero and CMOD equal to $d, L_{d}(f)$ is signal 608 loss due to crack. $L_{d}(f)$ is calculated from equation (4) and (5) as,

$609 \quad L_{d}(f)=\left(\frac{r_{s}(f)_{d}}{r_{s}(f)_{0}}\right)$

610 The attenuation factor, $A(f)$ was determined as the average of $L_{d}(f)$ at the bandwidth

$611 \quad A(f)=\operatorname{Avg} \cdot\left(L_{d}(f)\right)_{f_{1}, f_{2}}$

612 where the magnitudes of FFT corresponding to a frequency $f_{1}$ to $f_{2}$ at amplitude equal to $\frac{1}{\sqrt{2}}$ 613 of peak amplitude in the FFT (Figure 16b).

614 Attenuation factor $(A(f))$ was calculated for all the received signals using Equation $(7)$ and is 615 shown in Figure 17a. There is no change in the attenuation factor even at very large crack 616 openings for the transmission paths which do not encounter crack. There is a considerable 617 decrease in the $A(f)$ for the transmission paths which encounters the crack, even at the stress618 induced physical opening of $12 \mu \mathrm{m}$ at the mid-height of the beam. The decrease in the 619 attenuation factor values were high for signals received from PZTs placed at the soffit of the 620 beam when compared with values of the signals received at the PZTs place at the mid-height 621 of the beam. This is can be explained considering the crack opening in the direct stress wave 622 path between an AR pair. The crack width opening along the depth of a specimen at different 623 values of GMOD are shown in Figure 10b. The signals received at the PZTs placed at the 624 soffit of the beam experienced a higher attenuation because of a larger physical opening in the 
625

626

627

628

629

630

631

632

633

634

635

636

637

638

639

640

641

642

643

direct path connecting the actuator to the receiver. Upon unloading, the physical discontinuity is larger in the stress wave path connecting PZT patches located on the beam soffit from actuators located across the crack. There is a complete attenuation of direct stress wave when the stress induced crack opening reaches a value of $100 \mu \mathrm{m}$.

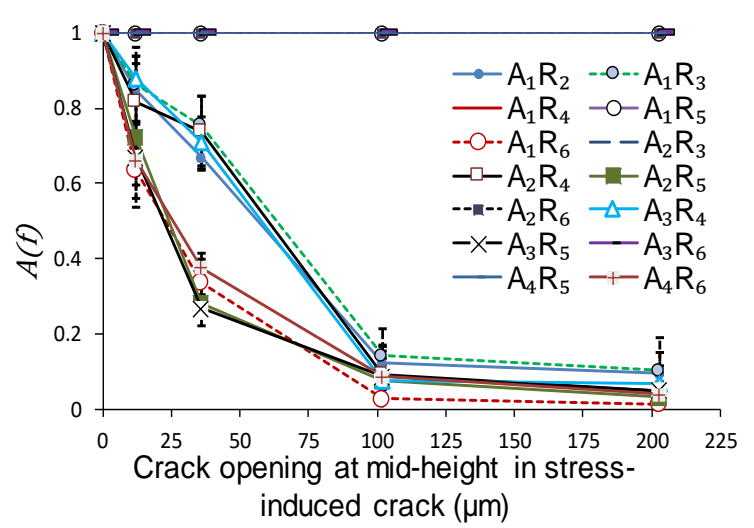

(a)

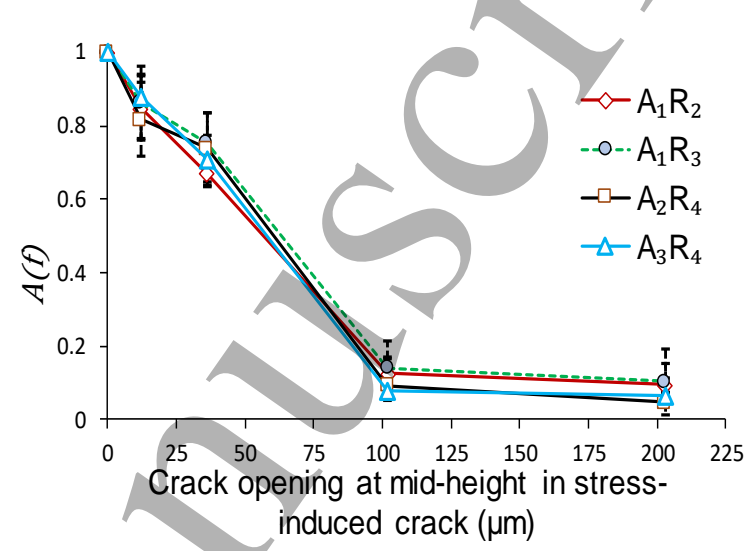

(b)

Figure 17. Attenuation factor $(A(f))$ as a function of crack opening at mid-height in the stress induced crack (a) all AR pairs; (b) For AR pairs placed at the mid-height of the beam.

The changes in attenuation factor corresponding to the transmission paths which encounter a physical opening produced by a stress-induced crack and pass through center line of beam (actuator and receiver located in the mid-height of the beam) are shown in Figure 17b. For the PZTs mounted at the mid-height, the $A(f)$ is identical for the stress wave paths linking the AR pair which cross the crack plane. It can therefore be concluded that the observed $A(f)$ in these cases is only due to the magnitude of the physical discontinuity produced by the crack. The changes in $A(f)$ are only due to the changes in characteristics of discrete crack such as depth and width of crack, irrespective of length of signal transmission path. The similarity in the trends of changes in attenuation factor irrespective of length of signal transmission path also 
644 suggests that the attenuation factor only depends on the severity of crack irrespective of all 645 other intervening effects. The $A(f)$, therefore provides an effective way to analyze the received 646 signals to detect changes introduced by material discontinuity in the stress wave path. The $647 A(f)$ is sensitive to a physical discontinuity in the concrete medium even in the unloaded state 648 associated with a stress induced crack opening on the order of $10 \mu \mathrm{m}$. The physical 649 discontinuity in the concrete associated with a stress-induced crack opening on the order of $650100 \mu \mathrm{m}$ produces a complete attenuation of the stress wave. This indicates that there is no 651 direct transmission of direct stress waves centered on $120 \mathrm{KHz}$ across a physical discontinuity 652 in concrete resulting from a stress-induced opening between the crack faces on the order of $6530.1 \mathrm{~mm}$.

654 A damage index derived from the RMSD is used to calculate the difference between the 655 conductance signatures recorded for different crack openings. The RMSD with respect to the 656 baseline measurement (zero CMOD) were calculated in the frequency range of the bandwidth 657 for first and second resonant peaks using Equation (8), where, $x_{i}$ and $y_{i}$ are the values of 658 baseline conductance and conductance at different CMOD levels and $\mathrm{N}$ is total number of 659 frequencies in the bandwidth of the corresponding peak.

$660 \quad \operatorname{RMSD}=\sqrt{\frac{\sum_{i=1}^{N}\left(y_{i}-x_{i}\right)^{2}}{\sum_{i=1}^{N}\left(x_{i}\right)^{2}}}$

661 Damage index based on RMSD for PZTs of different positions are is shown in Figure 18a.

662 The damage index was calculated using the frequency range of the bandwidth for second 663 resonant peak.PZT3, which lócated $180 \mathrm{~mm}$ from the crack location registered a small change 664 in the RMSD with increasing crack opening. PZT2 and PZT5, which are attached on the face 665 and the soffit, respectively at the distances of $50 \mathrm{~mm}$ from the beam centerline show an 666 increase in the RMSD with increasing CMOD. Figure 18b shows the RMSD calculated using 667 first resonance peak in the frequency range of bandwidth. PZT3 is showing same RMSD trend 
668

669

670

671

672

673

674

675

676

677

678

679

680

681

682

683

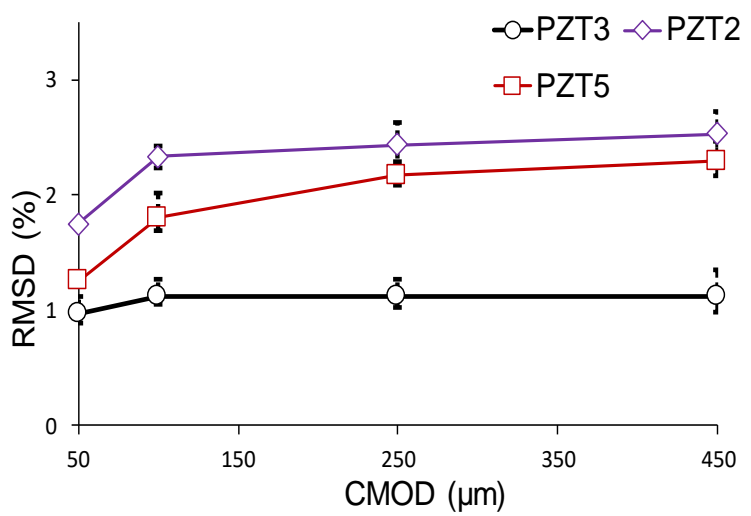

(a) PZT.

as for second peak. There is a larger increase in value for RMSD at all CMOD values for PZT2 and PZT5 when comparing with RMSD of the second peak. The scatter in the RMSD values may be attributed to the irregular trend in the local peaks present on first resonance peak of the

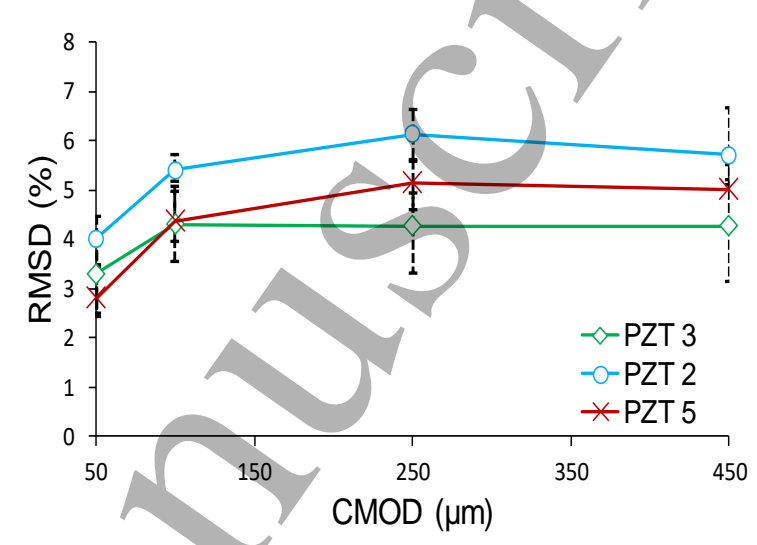

(b)

Figure 18. RMSD (\%) (a) Second resonance peak (b) First resonance peak

The measured RMSD detects changes in the first cycle while the changes in the subsequent load cycles are not significant. The RMSD measurements are indicative of changes in the material medium within the zone of influence produced by the discontinuity introduced by the crack. The discontinuity in the unloaded state represents the physical separation introduced in the material due to the stress-induced crack opening. The RMSD values indicate that the EI measurement is very sensitive in detecting the discontinuity more than the magnitude of the discontinuity. EI measurement would therefore provide a very sensitive measure of crack initiation in concrete in the vicinity of the PZT patch.

685

\section{6. Summary and Findings}


687 A combined local and distributed monitoring system for concrete structures using an array 688 of surface mounted PZT patches is presented. EI impedance measurements from individual 689 PZT patches are used for local monitoring while the through-transmission stress wave 690 propagation technique is used for distributed sensing. Progression of discrete crack in concrete 691 was evaluated using a full-field displacements measured on the surface of the beam obtained 692 with the use digital image correlation and was correlated with measurements obtained from the 693 PZT patches. The crack opening is mapped from a very small value on the order to $10 \mu \mathrm{m}$ to $694100 \mu \mathrm{m}$. The material away from the localized discontinuity produced by the crack is shown to relatively free from any damage.

Both the EI and the stress-wave propagation techniques are shown to be influenced by discontinuity in the concrete substrate produced by a stress-induced crack. The EI measurement is sensitive to the formation of localized damage in the form of a load induced crack in the vicinity of the PZT patch. A new damage index known as attenuation factor is introduced for the wave propagation technique. The attenuation factor is shown to be an effective damage index for detecting the severity of discontinuity produced by a crack. The attenuation factor is shown to determine attenuation of the wave produced by the discontinuity encountered by the direct stress wave, independent of the length of propagation. The attenuation measurements were performed in the unloaded state, where the removal of the load results in closing of the crack. The through transmission of direct stress wave through the medium is very sensitive to the presence of a discontinuity left in the medium resulting from a stress-induced crack in its path. There is an attenuation of the wave even for a physical discontinuity in the concrete in the stress free state associated with a small stress-induced crack opening on the order of $10 \mu \mathrm{m}$. There is a complete attenuation of the direct stress wave transmission at $120 \mathrm{kHz}$ for physical discontinuity in the concrete associated with a stress-

711 induced/crack opening on the order of $100 \mu \mathrm{m}$. 
712 The measurements from the PZT patches indicate that the localized discontinuity in 713 concrete produced by a stress induced crack is easily detected using the attenuation factor from 714 the direct stress wave transmission path. The stress-induced crack has considerable crack 715 closing stresses provided by aggregates bridging the crack. The discontinuity in the unloaded 716 state represents the physical separation introduced in the material due to the stress-induced 717 crack opening. The cracks which would have propagated due to overloads, but are closed due 718 to removal of the loads can therefore be easily be detected using attenuation factor 719 measurements. This provides a very convenient measure for monitoring increment of damage 720 due in the material. Further, crack opening on the order of $200 \mu \mathrm{m}$ under service loads is often 721 stipulated for durable design [ACI 224R-01 (2001), IS-456 (2000)]. The attenuation factor 722 from distributed sensing provides for monitoring discontinuity in the material medium even in 723 the unloaded state, when the crack is not visually detectable. The initiation of the discontinuity 724 would be reflected in the EI signature if the discontinuity lies within its zone of influence. 725 Service load condition of the concrete structure can therefore be monitored conveniently using 726 combined EI and AR modes of an array of PZT patches.

\section{References}

729 [1] Achenbach, J.D., Komsky, I.N., Lee, Y.C. and Angel, Y.C., (1992), "Self-calibrating 730 Ultrasonic Technique for Crack Depth Measurement," Journal of Nondestructive 731 Evaluation, Vol.11, No.2, pp.103-108.

732 [2] ACI-224R-01.,(2001), "Control of Cracking in Concrete Structures," Farmington Hills, 733 MI: American Concrete Institute. 
734 [3] Aggelis, D.G. and Shiotani, T., (2007), "Experimental Study of Surface Wave 735 Propagation in Strongly Heterogeneous Media," The Journal of the Acoustical Society 736 of America, Vol. 122, No. 5, pp.151-157.

737 [4] Bhalla S, and Soh CK (2004), "Structural Health Monitoring by Piezo-Impedance Transducers. I: Modeling,” Journal of. Aerospace. Engineering, Vol. 17, No. 4, pp.154 165.

[5] Bruck, H.A., McNeill, S.R., Sutton, M.A, and Peters, W.H., (1989), "Digital Image Correlation Using the Newton Raphson Method of Partial Differential Correction," Experimental Mechanics, Vol. 29, No. 3, pp. 261-267.

[6] Divsholi, B.S. and Yang, Y., (2014), "Combined Embedded and Surface-bonded Piezoelectric Transducers for Monitoring of Concrete/Structures," NDT \& E International, Vol. 65, pp.28-34.

[7] Gali, S. and Subramaniam, K.V., (2018), "Multi-linear Stress-Crack Separation Relationship for Steel Fiber Reinforced Concrete: Analytical Framework and Experimental Evaluation," Theoretical and Applied Fracture Mechanics, Vol. 93, pp.3343.

[8] Giurgiutiu, V. and Rogers, C.A., (1999), "Modeling of the Electro-mechanical (E/M) Impedance Response of a Damaged Composite Beam,”. Adaptive Structures and Material Systems- November 14-19, pp.39-46.

[9] Giurgiutiu, V., (2001), "Structural Health Monitoring with Piezoelectric Active Sensors," Academic Press, Oxford, UK.

[10] Huo, L., Chen, D., Liang, Y., Li, H., Feng, X. and Song, G., (2017), “Impedance Based Bolt Pre-Load Monitoring Using Piezoceramic Smart Washer," Smart Materials and Structures, Vol. 26, No.5, p.057004.

758 [11] I.S 456: 2000, "Indian Standard-Plain and Reinforced Concrete Code of Practice". 
759 [12] Jung, Y.C., Kundu, T. and Ehsani, M.R., (2002), “A New Nondestructive Inspection

760

761

762

763

764

765

766

767

768

769

770

771

772

773

774

775

776

777

778

779

780

781

782 Technique for Reinforced Concrete Beams,” ACI Materials Journal, Vol. 99, No. 3, pp.292-299.

[13] Kee, S.H. and Nam, B., (2015), “Automated Surface Wave Measurements for Evaluating the Depth of Surface-Breaking Cracks in Concrete," International Journal of Concrete Structures and Materials, Vol. 9, No. 3, pp.307-321.

[14] Liang, C., Sun, F.P., and Rogers, C.A., (1994), “An Impedance Method for Dynamic Analysis of Active Material Systems," Journal of Vibration and Acoustics, Vol.116, No.1, pp. 120-128.

[15] Liang, Y., Li, D., Parvasi, S.M., Kong, Q. and Song, G., (2016), “Bond-slip detection of concrete-encased composite structure using electro-mechanical impedance technique," Smart Materials and Structures, Vol.25, No.9, p.095003.

[16] Lim, Y Y., and Soh, C K., (2012), "Effect of varying axial load under fixed boundary condition on admittance signatures of electromechanical impedance technique,’ Journal of Intelligent Material Systems and Structures, doi: 10.1177/1045389X12437888.

[17] Lu, Y., Li, J., Ye, L. and Wang, D., (2013), "Guided Waves for Damage Detection in Rebar-reinforced Concrete Beams," Construction and Building Materials, Vol. 47, pp.370-378.

[18] Luo, M., Li, W., Hei, C. and Song, G., (2016), “Concrete Infill Monitoring in ConcreteFilled FRP Tubes Using a PZT-Based Ultrasonic Time-of-Flight Method," Sensors, Vol. 16, No. 12, p.2083.

[19] Memmolo, V., Maio, L., Boffa, N.D., Monaco, E. and Ricci, F., (2016), "Damage Detection Tomography Based on Guided Waves in Composite Structures Using a Distributed Sensor Network,” Optical Engineering, Vol. 55, No.1, pp.011007-011007. 
783 [20] Moradi-Marani, F., Rivard, P., Lamarche, C.P. and Kodjo, S.A., (2014), "Evaluating the 784

[21] Na, S. and Lee, H.K., (2012), “A technique for improving the damage detection ability of the electro-mechanical impedance method on concrete structures," Smart Materials and Structures, Vol.21, No.8, p.085024.

[22] Narayanan, A., and Subramaniam, K V L., (2016a), "Experimental evaluation of loadinduced damage in concrete from distributed microcracks to localized cracking on electro-mechanical impedance response of bonded PZT”, Construction and Building Materials, Vol.105, pp. 536-544.

[23] Narayanan, A., and Subramaniam, K. V. L., (2016b), "Sensing of Damage and Substrate Stress in Concrete Using Electro-Mechanical Impedance Measurements of Bonded PZT Patches", Smart Materials and Structures, Vol. 25, No. 9, p.095011.

[24] Narayanan, A., Kocherla, A. and Subramaniam, K.V., (2017), "Embedded PZT Sensor for Monitoring Mechanical Impedance of Hydrating Cementitious Materials," Journal of Nondestructive Evaluation, Vol. 36, No. 4, p.64.

[25] Narayanan, A., Kocherla, A. and Subramaniam, K.V.L., (2018), "Understanding the Coupled Electromechanical response of PZT attached to Concrete: Influence of Substrate size," under review in Measurement.

[26] Park, G., Cudney, H., and Inman, D., (2000), "Impedance-Based Health Monitoring of Civil Structural Components," Journal of Infrastructure Systems, Vol.6, No.4, pp. 153160.

[27] Park, S., Ahmad, S., Yun, C.B. and Roh, Y., (2006), "Multiple Crack Detection of Concrete Structures Using Impedance-based Structural Health Monitoring Techniques,” Experimental Mechanics, Vol. 46, No.5, pp.609-618. 
808 [28] Reddy, K.C. and Subramaniam, K.V., (2017), "Analysis for multi-linear stress-crack opening cohesive relationship: Application to macro-synthetic fiber reinforced concrete," Engineering Fracture Mechanics, Vol. 169, pp.128-145.

[29] Rucka, M. and Wilde, K., (2013), "Experimental Study on Ultrasonic Monitoring of Splitting Failure in Reinforced Concrete," Journal of Nondestructive evaluation, Vol. 32 , No. 4, pp.372-383.

[30] Schreier, H, and Sutton, M., (2002), "Systematic Errors in Digital Image Correlation Due to Undermatched Subset Shape functions," Experimental Mechanics, Vol.42, No.3, pp. 303-310.

817 [31] Song, F., Huang, G.L., Kim, J.H. and Haran, S., (2008), "On the Study of Surface Wave Propagation in Concrete Structures Using a Piezoelectric/Actuator/Sensor System,”. Smart Materials and Structures, Vol. 17, No. 5, p.055024.

[32] UNI 11039-2: 2003, "Steel Fibre Reinforced Concrete - Test Method for Determination of First Crack Strength and Ductility Indexes. National Italian Unification Centre,’, Italy.

[33] Wang, X. and Subramaniam, K.V., (2011), "Ultrasonic Monitoring of Capillary Porosity and Elastic Properties in Hydrating Cement Paste," Cement and Concrete Composites, Vol.33, No.3, pp.389-401.

[34] Watanabe, T., Trang, H.T.H., Harada, K. and Hashimoto, C., (2014), "Evaluation of Corrosion-Induced Crack and Rebar Corrosion by Ultrasonic Testing," Construction and Building Materials, Vol.67, pp.197-201.

[35] Xu, B., Chen, H. and Xia, S., (2017), "Numerical Study on The Mechanism of Active Interfacial Debonding Detection for Rectangular CFSTs Based on Wavelet Packet Analysis with Piezoceramics," Mechanical Systems and Signal Processing, Vol. 86, pp.108-121. 
832 [36] Xu, Y.G., and Liu, G.R., (2002), "A Modified Electro-Mechanical Impedance Model of 833 Piezoelectric Actuator-Sensors for Debonding Detection of Composite Patches," Journal 834 of Intelligent Material Systems and Structures, Vol. 13, No. 6, pp. 389-396.

835 [37] Zagrai, A.N. and Giurgiutiu, V., (2001), "Electro-Mechanical Impedance Method for 836 Crack Detection in Thin Plates,” Journal of Intelligent Material Systems and Structures, 837 Vol. 12, No. 10, pp.709-718.

838 [38] Zhu, J. and He, L., (2011), "Study on Piezoelectric Wave Propagation Based 839 Nondestructive Monitoring Method of Concrete,” In IEEE International Conference on 840 Electric Technology and Civil Engineering (ICETCE), pp. 764-767. 